ESAIM: M2AN 47 (2013) 987-1016

DOI: $10.1051 / \mathrm{m} 2 \mathrm{an} / 2012054$
ESAIM: Mathematical Modelling and Numerical Analysis

www.esaim-m2an.org

\title{
A PREDiCTive Method Allowing THE USE OF A SINGLE IONIC MODEL IN NUMERICAL CARDIAC ELECTROPHYSIOLOGY
}

\author{
M. Rioux ${ }^{1}$ AND Y. BOURGAulT ${ }^{1}$
}

\begin{abstract}
One of the current debate about simulating the electrical activity in the heart is the following: Using a realistic anatomical setting, i.e. realistic geometries, fibres orientations, etc., is it enough to use a simplified 2-variable phenomenological model to reproduce the main characteristics of the cardiac action potential propagation, and in what sense is it sufficient? Using a combination of dimensional and asymptotic analysis, together with the well-known Mitchell-Schaeffer model, it is shown that it is possible to accurately control (at least locally) the solution while spatial propagation is involved. In particular, we reduce the set of parameters by writing the bidomain model in a new nondimensional form. The parameters of the bidomain model with Mitchell-Schaeffer ion kinetics are then set and shown to be in one-to-one relation with the main characteristics of the four phases of a propagated action potential. Explicit relations are derived using a combination of asymptotic methods and ansatz. These relations are tested against numerical results. We illustrate how these relations can be used to recover the time/space scales and speed of the action potential in various regions of the heart.
\end{abstract}

Mathematics Subject Classification. 34C15, 35B40, 78A70, 92C50.

Received January 5, 2012.

Published online 7 June 2013.

\section{INTRODUCTION}

Numerical modelling is a main tool for understanding the propagation of electrophysiological waves in healthy and pathological hearts. There is great need in developing prediction tools that could be used for example to identify the mechanisms behind alternans and arrhythmias, develop control strategies for reducing the impact of conduction pathologies and improve therapy planning [6]. For the last decade or so, there is an increasingly popular trend to patient-specific modelling, where the predictions in clinical applications rely on the personalisation of cardiac electrophysiology models. For instance, mapping models are used in $[27,28]$ to estimate the model parameters, resulting in model predictions similar to the actual clinical data.

A first crucial step in setting simulations in cardiac electrophysiology consists in choosing appropriate ionic models, typically systems of ordinary differential equations (ODE) able to reproduce the fast and slow dynamics of the action potential (AP), i.e. the excitation of cardiac cells through time. The ionic models available are

Keywords and phrases. Asymptotic analysis, cardiac electrophysiology, Mitchell-Schaeffer model.

1 Department of Mathematics and Statistics, University of Ottawa, Canada. ybourg@uottawa.ca 
numerous (see www.cellML.org) and it is important to understand the benefits and the limitations of each model so that an appropriate choice can be made [7]. Ionic models can be split in three classes respective to the way these models are constructed: the physiological cell ionic models (e.g. Luo-Rudy [21], Beeler-Reuter [3], Hodgkin-Huxley $[15,25]$ ), the reduced ionic models (e.g. the 3 -variables Fenton-Karma model is a reduction of the Luo-Rudy 1 model [12]), and the phenomenological models (e.g. FitzHugh-Nagumo (FHN) [13,24], AlievPanfilov [1], Mitchell-Schaeffer [11,22]). Accordingly, these three types of models are intended to reproduce different phenomena. See [7] for a good review.

With any of these types of ionic models, a main problem arises when one wants to incorporate the ionic model in a model for spatial propagation: the time and space scales have to match and some parameters have to be tuned properly. The ionic models are particularly sensitive to the variation of the parameters. It is hard to change these parameters so that the solution of the differential equations reacts with the right time and space scales. As a result, doing realistic simulations requires that the influence of each parameter involved has to be well understood.

This overall understanding is hard to achieve and to address this difficulty we propose to reduce the set of parameters in the equations. Phenomenological models become a natural choice, a framework adapted for most applications where it may not be necessary to construct a highly detailed and computationally expensive physiological ionic model [7]. The Mitchell-Schaeffer model could be used to reproduce the action potential dynamics.

To reduce further the set of parameters in the equations, a nondimensionalisation is performed and much less parameters are necessary that is to say a single dimensionless number replaces all parameters related to the propagation of the potential. Using an asymptotic analysis, a bijective relation between the set of the ionic model parameters and the set of solution features is built. The combination of the dimensional analysis and the asymptotic analysis makes possible to set precisely the parameters of the ionic model (electrical activity of a single cardiomyocyte) and the bidomain models (electrical activity propagated throughout the surrounding heart tissues).

Aside from its theoretical interest, this work proposes steps to control the wave's shape and its propagation in a region of local constant conductivity. If the use of the Mitchell-Schaeffer model turns out to be sufficient, every model parameter can be chosen properly by virtue of the knowledge of the resulting solution (the approach becomes predictive). Remark that a similar approach could probably be successfully applied to another ionic model. This is a huge step toward the direct computation of the electrocardiogram, where the model parameters are properly tuned in different regions of the heart and the cardiac AP is computed and propagated through a heart-thorax geometry. This could lead to an alternative way for exploring the inverse problem: simulate the electrode acquisition process, solve the inverse problem, and validate with a perfectly known source.

As a last comment, this work results from a combination of methods that have been used extensively. The difference between the previous works in this domain ([5,16] for ODE models and $[22,29,30]$ for mapping models) is that here, the results of the analysis not only portrays a general behaviour of the solution features (i.e. give some dependences on some parameters of the model, but not all possibly influent parameters), but can reliably predict a desired solution with explicit dependences on all model parameters and all the physiological constants. Our approach is able to deal with isolated as well as sequences APs, but it also can be used to precisely shape propagated APs. This approach is then suitable for predictions.

The article is outlined as follows. Section 2 presents background material on AP and its modelling through ionic and bidomain models. Section 3 proposes a new way to nondimensionalise the bidomain model and reduce the set of parameters. Section 4 contains the main results of this article. The parameters of the bidomain model with Mitchell-Schaeffer ion kinetics are set and shown to be in one-to-one relation with the main characteristics of the four phases of a propagated AP. Explicit relations are derived using a combination of asymptotic methods and ansatz. These relations are tested against numerical results. Section 5 illustrates how these relations can be used to recover the main characteristics of the AP in various regions of the heart. Section 6 concludes with general remarks. 


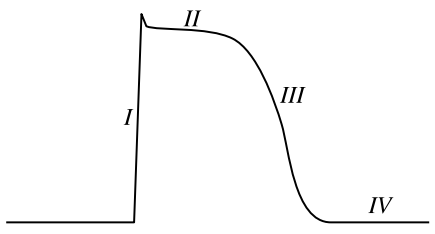

FiguRE 1. The transmembrane potential versus time with the four main phases of the cardiac AP. This is the typical shape of a ventricular AP.

\section{BACKGROUND MATERIAL}

For electrically excitable cells, an action potential (AP) is a biological manifestation described by a fast rise (depolarisation) and fall (repolarisation) of the transmembrane potential, where the transmembrane potential is defined as the difference between the intracellular and the extracellular potentials. The AP of a cell is the result of the movement of ions through the cell membrane (passive transport and active transport e.g. voltage-gated channels), and this electrical activity is stimulated typically by the electrical activation from adjacent cells. The resulting AP propagates in the myocardium and subsequently follows the contraction of the heart, allowing blood to be pumped throughout the body. An efficient contraction is ensured by a well-regulated stimulation, hence it is crucial to understand the inherent physiology.

If a heart beat results from the complex interaction of different APs in different portions of the heart, the ventricular AP reveals far enough to have a good overall understanding of the cardiac AP. Here is a brief description (see [5]) of the ventricular AP featuring the most important four phases. Figure 1 shows a typical ventricular AP separated in its four phases.

Phase I. Transmembrane potential upstroke or depolarisation (short time scale): The transmembrane potential is initially at its resting state $\left(V_{\text {rest }} \simeq-85 \mathrm{mV}\right)$, where all the fast $\mathrm{Na}^{+}$channels are closed. A partial depolarisation opens these channels causing a large influx of $\mathrm{Na}^{+}$ions, further increasing the depolarisation. The cell gets positively charged $\left(V_{\max } \simeq 40 \mathrm{mV}\right)$ or depolarised.

Phase II. Excited phase (long time scale): An outward current of potassium ions is balanced by an inward movement of calcium ions, causing a plateau. Sodium ions are still flooding in and just about keep pace. The transmembrane potential falls slowly. Note: The transient net outward current causing the small downward deflection (such an overshoot is observed in some cardiomyocyte APs) is mainly due to the movement of $\mathrm{K}^{+}$ions.

Phase III. Downstroke or repolarisation (short time scale): The calcium channels close while potassium channels are still open. The net outward positive current causes the cell to repolarise until the transmembrane potential is restored to about $-85 \mathrm{mV}$. The cell may pass its equilibrium polarisation, and becomes hyperpolarised.

Phase IV. Recovery period (long time scale): At the beginning of this period, the transmembrane potential gets close to its resting state, but some gates or ionic currents have not yet reached their equilibrium value. The recovery phase ends when all the currents and gates reach the equilibrium state within a prescribed tolerance or threshold. We abstain from using the expression "refractory period" and stick to "recovery period" while connections may be made between these notions given that partial recovery leads to absolute or relative refractoriness.

In order to obtain integrative anatomically accurate models for the AP, one needs to gather information from many sources. Collecting information can become a huge task just to characterise a healthy human heart not to mention when heart abnormalities are studied [16,32]. Moreover, depending in which tissue the AP is propagated, the cells are different and so are the features of the AP (see Tab. 1). Throughout the text, the term solution feature is taken as any measure characterising the solution of a model describing the AP. For instance 
TABLE 1. Speeds and absolute refractory periods (ARP) are taken from [20] where the heart cycle lasts $700 \mathrm{~ms}$. Similar values for speeds can also be found at http: ://cvphysiology.com/ Arrhythmias/A003.htm

\begin{tabular}{|l|c|c|}
\hline Tissue & $\begin{array}{c}\text { Speed } \\
{[\mathrm{cm} / \mathrm{s}]}\end{array}$ & $\begin{array}{c}\text { ARP } \\
{[\mathrm{ms}]}\end{array}$ \\
\hline Ventricle & $35-55$ & $255-275$ \\
Atria & 60 & $250-270$ \\
SA node & 5 & 430 \\
AV node & 5 & 300 \\
Bundle of His & 170 & 210 \\
Bundle Branches & 200 & $270-310$ \\
Purkinje fibres & 180 & 320 \\
\hline
\end{tabular}

the durations of phases and the speed of the propagated potential wavefront are solution features that are going to be studied.

\subsection{The model for propagation}

Propagation of the action potential is commonly modelled through the bidomain model or an approximation of this model called the monodomain model (see [16,32] and references therein). The bidomain model represents the myocardium as an homogenised continuum of the myocyte intracellular and extracellular spaces, with an electrical potential for both spaces existing at every point of the myocardium. The heart covers the domain $H$ with boundary $\partial H$ separating the myocardium from the cardiac cavities and the thorax. Under the assumption that the heart is isolated from its surrounding (e.g. non-coupled with the thorax), solving the bidomain model consists in finding $\left(u, v, u_{e}\right)$ such that

$$
\begin{array}{rcc}
\frac{\partial v}{\partial t}=g(u, v) & \text { in } & H, \\
\chi\left(C_{\mathrm{m}} \frac{\partial u}{\partial t}+I_{\text {ion }}(u, v)\right)=\nabla \cdot\left(\sigma_{i} \nabla u\right)+\nabla \cdot\left(\sigma_{i} \nabla u_{e}\right) & \text { in } & H, \\
\nabla \cdot\left(\sigma_{i} \nabla u\right)+\nabla \cdot\left(\left(\sigma_{i}+\sigma_{e}\right) \nabla u_{e}\right)=0 & \text { in } & H, \\
n_{H} \cdot\left(\sigma_{e} \nabla u_{e}\right)=0 & \text { on } & \partial H, \\
n_{H} \cdot\left(\sigma_{i} \nabla u_{i}\right)=0 & \text { on } & \partial H
\end{array}
$$

where $v$ is the vector of variables for the ionic model; $u_{i}, u_{e}$ and $u=u_{i}-u_{e}$ are, respectively, the intracellular, extracellular and transmembrane potentials; $\chi$ is the cell membrane surface-to-volume ratio; $C_{\mathrm{m}}$ is the specific capacitance (per unit area) of the cell membrane; $\sigma_{i}$ and $\sigma_{e}$ are, respectively, the intracellular and extracellular conductivity tensors.

The first equation represents the system of equations of the ionic model, which is coupled with the bidomain model via the current $I_{\text {ion }}$. For a simple 2-variable phenomenological model, the reaction term $I_{\text {ion }}$ is expressed as an algebraic function $f(u, v)$. The second equation models the entry of ions into the intracellular space, while the third equation represents the conservation of ionic charges in the extracellular space. The fourth and fifth equations express the fact that conduction cannot occur through the boundary $\partial H$ both from the extracellular and the intracellular spaces.

\subsection{The choice of the ionic model}

The Mitchell-Schaeffer (MS) model was first introduced in [11]. This 2-variable phenomenological model has several qualities that makes it a candidate of choice for achieving our goal of matching several AP features (conduction speed, time scales, restitution) with the simplest possible model. First of all, because it is derived 
from the Fenton-Karma ionic model, it has benefits of an ionic model though it is a phenomenological model. Moreover, many authors have used the MS model for realistic clinical applications. In order to simulate a right bundle-branch block Boulakia and et al. [4] used the MS model on the one hand to keep as low as possible the complexity of the model and on the other hand to model as accurately as possible the physical phenomena. As an other example, mapping models for predictions of the excited phase duration and the recovery duration (derived in $[11,22]$ ) were proved to be efficient for cardiac model personalisation using real patient data in $[27,28]$.

The original MS model reads as

$$
\begin{aligned}
& \frac{\mathrm{d} u}{\mathrm{~d} t}+f(u, v)=0, \text { where } f(u, v)=\frac{1}{\tau_{\text {in }}} v u^{2}(u-1)+\frac{1}{\tau_{\text {out }}} u \\
& \frac{\mathrm{d} v}{\mathrm{~d} t}=g(u, v), \text { where } g(u, v)= \begin{cases}\frac{1}{\tau_{\text {open }}}(1-v) & \text { for } u<u_{\text {gate }}, \\
-\frac{1}{\tau_{\text {close }}} v & \text { for } u \geq u_{\text {gate }},\end{cases}
\end{aligned}
$$

with $\tau_{\text {in }}=0.3 \mathrm{~ms}, \tau_{\text {out }}=6 \mathrm{~ms}, \tau_{\text {open }}=120 \mathrm{~ms}, \tau_{\text {close }}=150 \mathrm{~ms}$ and $u_{\text {gate }}=0.13$, a set of parameter values proposed in [22].

Several modified versions of the MS model are brought in the literature (see for example $[10,17,29,30]$ ). The following outlines the subtleties of the different versions of the reaction terms.

A first variant of the MS model is presented in [10]. A noticeable difference between the original MS and this modified version is the introduction of the parameter $a$ in $f(u, v)$

$$
f_{\text {pace }}(u, v)=\frac{1}{\tau_{\text {in }}} v(u+a)^{2}(u-1)+\frac{1}{\tau_{\text {out }}} u,
$$

where $a=0.02$ is suggested. It can be used to control the excitability and mimic a natural pacemaker activity.

Schaeffer and et al. $[29,30]$ brought an extension to the original MS model. The new model has three variables, where the third variable is concentration-like and acts as a memory variable. It also helps the charge balance through time. This model improves predictions where rate dependence and accommodation are involved (see $[29,30]$ for details). Besides this, a relevant change is brought to the source term $g(u, v)$ upon what the solution features the overshoot after the depolarisation as illustrated in Figure 1. The closing rate $\tau_{\text {close }}$ becomes voltage-dependent and reads as

$$
\frac{1}{\tau_{\text {close }}(u)}= \begin{cases}\frac{1}{\tau_{\text {fclose }}}-\left(\frac{1}{\tau_{\text {fclose }}}-\frac{1}{\tau_{\text {sclose }}}\right) \frac{1-u}{1-u_{\text {sldn }}} & \text { if } u>u_{\text {sldn }} \\ \frac{1}{\tau_{\text {sclose }}} & \text { otherwise }\end{cases}
$$

where $\tau_{\text {fclose }}$ and $\tau_{\text {sclose }}$ are time scale parameters referring respectively to a fast and slow closing rate. Right after the depolarisation, the closing rate slows down progressively until $u=u_{\text {sldn }}$. Past this value the rest of the phase II happens as with the original MS model. In [30], the following set of parameters is proposed: $\tau_{\text {in }}=0.28 \mathrm{~ms}, \tau_{\text {out }}=3.2 \mathrm{~ms}, u_{\text {gate }}=0.13, u_{\text {sldn }}=0.89, \tau_{\text {open }}=500 \mathrm{~ms}, \tau_{\text {sclose }}=22 \mathrm{~ms}$ and $\tau_{\text {fclose }}=320 \mathrm{~ms}$.

In the text, what is called the modified MS model has the reaction terms $f(u, v)$ of equation $(2.8)$ and $g(u, v)$ of equation (2.7) with the associated time scale $\tau_{\text {close }}(u)$ of equation (2.9). In instances where the overshoot following depolarisation is not needed, one can set $\tau_{\text {close }}(u) \equiv \tau_{\text {sclose }}$ and recovers the original MS model if $a=0$.

\section{Nondimensionalisation}

We first proceed with a nondimensionalisation of the problem (2.1)-(2.5), an essential ingredient behind any asymptotic analysis. A main issue comes from the fact that the bidomain model takes a dimensional form but is 
coupled with nondimensional ionic models. Indeed a common way found in the literature for writing the source terms describing the transmembrane current of a specific ion is given by

$$
I_{\mathrm{ion}}=\frac{h p(u, v)}{\tau}
$$

where $h$ is a gating variable, $p(u, s)$ is a function of the transmembrane potential and $\tau$ is the time scale of this charge flow. As $p(u, s)$ can be any function (combination of exponentials, polynomials, etc.), the variables $h, u$ and $v$ are often taken dimensionless and $\tau$ dimensional (in seconds) in order to preserve the unit balance in the equations. A consequence of using nondimensional potentials is that the source terms of ionic models proposed in the literature are often expressed in $s^{-1}$ to match the units of the terms $\partial v / \partial t$ or $\partial u / \partial t$ in the conduction model.

To perform the nondimensionalisation and unit-balancing of the dimensional bidomain model for an isolated heart (2.1)-(2.5), we define dimensionless variables and parameters, denoted with a $\sim$. The dimensional transmembrane potential $u$ is rescaled using $u=V_{\mathrm{m}} \widetilde{u}+V_{\text {rest }}$ with $V_{\mathrm{m}}=V_{\max }-V_{\text {rest }}$, where $V_{\mathrm{m}}, V_{\max }$ and $V_{\text {rest }}$ are, respectively, the characteristic action potential amplitude, the maximal potential attained once the cell is depolarised and the resting potential. The extracellular potential is also rescaled with $u_{e}=V_{\mathrm{m}} \widetilde{u}_{e}+V_{\text {rest }}$. For the independent variables, the time is rescaled with $t=\widetilde{t} T$ and the space with $x=\widetilde{x} L$. The eigenvalues of the conductivity tensor are rescaled with $\sigma_{\alpha}=\sigma \widetilde{\sigma}_{\alpha}$, with indices $\alpha=(i, l),(i, t),(e, l),(e, t)$, where $i$ and $e$ refer to the intracellular and extracellular medium respectively, $t$ and $l$ refer to the transverse and longitudinal eigendirections, both related to the fibre arrangement of the myocardium. Finally, $v$ is already taken nondimensional, in order to use directly the source terms given in the literature.

The following equations form the nondimensional version of the bidomain model coupled with any nondimensional ionic model.

$$
\begin{array}{rr}
\frac{\partial v}{\partial \widetilde{t}}=\widetilde{g}(\widetilde{u}, v) & \text { in } H, \\
\frac{\partial \widetilde{u}}{\partial \widetilde{t}}+\widetilde{I}_{\text {ion }}(\widetilde{u}, v)=N \widetilde{\nabla} \cdot\left(\widetilde{\sigma_{i}} \widetilde{\nabla}\left(\widetilde{u}+\widetilde{u_{e}}\right)\right) & \text { in } H, \\
\widetilde{\nabla} \cdot\left(\widetilde{\sigma_{i}} \widetilde{\nabla} \widetilde{u}\right)+\widetilde{\nabla} \cdot\left(\left(\widetilde{\sigma_{i}}+\widetilde{\sigma_{e}}\right) \widetilde{\nabla} \widetilde{u_{e}}\right)=0 & \text { in } H, \\
n_{H} \cdot\left(\widetilde{\sigma_{i}} \widetilde{\nabla} \widetilde{u}+\left(\widetilde{\sigma}_{i}+\widetilde{\sigma_{e}}\right) \widetilde{\nabla} \widetilde{u}_{e}\right)=n_{H} \cdot\left(\widetilde{\sigma}_{T} \widetilde{\nabla} \widetilde{u}_{T}\right) & \text { on } \partial H, \\
n_{H} \cdot\left(\widetilde{\sigma}_{i} \widetilde{\nabla} \widetilde{u}_{i}\right)=0 & \text { on } \partial H, \\
\widetilde{\nabla} \cdot\left(\widetilde{\sigma}_{T} \widetilde{\nabla} \widetilde{u}_{T}\right)=0 & \text { in } T, \\
n_{T} \cdot\left(\widetilde{\sigma}_{T} \widetilde{\nabla} \widetilde{u}_{T}\right)=0 & \text { on } \partial T .
\end{array}
$$

with $N=T \sigma / C_{\mathrm{m}} \chi L^{2}$. Note that $\widetilde{I}_{\text {ion }}(u, v)=\frac{T}{C_{\mathrm{m}} V_{\mathrm{m}}} I_{\text {ion }}(u, v)$ and $\widetilde{g}(u, v)=\frac{T}{V_{\mathrm{m}}} g(u, v)$. In general, the explicit versions of $I_{\text {ion }}$ and $g$ of equations (2.1)-(2.5) are useless because most of the phenomenological models are already written for nondimensional potentials. For the MS model or for any model with source terms of the form $(3.1)$, note that $\frac{1}{T} \widetilde{I}_{\text {ion }}(\widetilde{u}, v)=f(\widetilde{u}, v)$ with $f$ of equation $(2.6)$, and $\frac{1}{T} \widetilde{g}(\widetilde{u}, v)=g(\widetilde{u}, v)$ with $g$ of equation (2.7).

Remark 3.1. The dimensionless number $N$ can also be found with the Buckingham $\Pi$-theorem (see for example [23]). We have 5 physical variables $T, C_{\mathrm{m}}, V_{\mathrm{m}}, \sigma / \chi$ and $L$ expressible in terms of 4 independent fundamental units seconds $(s)$, Amperes $(A)$, Volts $(V)$ and meters $(m)$. The theorem says that any physically meaningful equation $\zeta\left(t, C_{\mathrm{m}}, V_{\mathrm{m}}, \sigma / \chi, L\right)=0$ is equivalent to an equation involving a single (\#variables - \#units $=1$ ) 
TABle 2. Parameters of the bidomain model for a human heart. Three different dimensional scalings are proposed. Adim1: based on the transmembrane potential upstroke. Adim2: based on the action potential duration and width. Adim3: based on convenient units. The values are taken from $[26,32]$.

\begin{tabular}{|c|c|c|c|c|c|}
\hline Description & Param. & $\begin{array}{l}\text { Values } \\
\text { Adim1 }\end{array}$ & $\begin{array}{l}\text { Values } \\
\text { Adim2 }\end{array}$ & $\begin{array}{l}\text { Values } \\
\text { Adim3 }\end{array}$ & Units \\
\hline Threshold potential & $V_{\mathrm{th}}$ & \multicolumn{3}{|c|}{-0.065} & $\mathrm{~V}$ \\
\hline Maximum potential & $V_{\max }$ & \multicolumn{3}{|c|}{0.040} & $\mathrm{~V}$ \\
\hline Resting potential & $V_{\text {rest }}$ & \multicolumn{3}{|c|}{-0.085} & $\mathrm{~V}$ \\
\hline Characteristic potential & $V_{\mathrm{m}}$ & \multicolumn{3}{|c|}{0.125} & $\mathrm{~V}$ \\
\hline Cell surface-to-volume ratio & $\chi$ & \multicolumn{3}{|c|}{$2 \times 10^{5}$} & $m^{-1}$ \\
\hline Transmembrane specific capacitance & $C_{\mathrm{m}}$ & \multicolumn{3}{|c|}{$1 \times 10^{-2}$} & $F / m^{2}$ \\
\hline Characteristic conductivity & $\sigma$ & \multicolumn{3}{|c|}{$1 \times 10^{-1}$} & $S / m$ \\
\hline Intracellular conductivity & $\tilde{\sigma}_{i, l}$ & \multicolumn{3}{|c|}{1.741} & - \\
\hline & $\tilde{\sigma}_{i, n}$ & \multicolumn{3}{|c|}{0.1934} & - \\
\hline Extracellular conductivity & $\tilde{\sigma}_{e, l}$ & \multicolumn{3}{|c|}{3.906} & - \\
\hline & $\tilde{\sigma}_{e, n}$ & \multicolumn{3}{|c|}{1.970} & - \\
\hline Characteristic time & $T$ & $1 \times 10^{-3}$ & 0.2 & $1 \times 10^{-3}$ & $s$ \\
\hline Characteristic length & $L$ & $5 \times 10^{-4}$ & 0.1 & $1 \times 10^{-3}$ & $m$ \\
\hline Dimensionless number & $N$ & 0.2 & $1 \times 10^{-3}$ & $5 \times 10^{-2}$ & - \\
\hline
\end{tabular}

dimensionless variable $N$ constructed from the original variables. We equivalently have $\zeta(N)=0$ with $N=T^{n_{1}} C_{\mathrm{m}}^{n_{2}} V_{\mathrm{m}}^{n_{3}}(\sigma / \chi)^{n_{4}} L^{n_{5}}$. Written in terms of fundamental units, the equation becomes

$$
1=(s)^{n_{1}}\left(A s /\left(V m^{2}\right)\right)^{n_{2}}(V)^{n_{3}}(A / V)^{n_{4}}(m)^{n_{5}} .
$$

The remaining problem consists in solving a homogeneous linear system of dimension 5 and as all the units are useful, the rank of the matrix involved is 4 . The dimension of the kernel of this matrix is the number of dimensionless numbers prescribed by the theorem (a single number here). Choosing one basis vector in the kernel, one gets the dimensionless number $N$ given in the text.

One is then left with a single dimensionless number $N$, and finding a value for it. The parameters $C_{\mathrm{m}}, V_{\mathrm{m}}, \chi$ and $\sigma$ are fixed, but the time scale $T$ and the space scale $L$ are still free. Fixing these is not necessarily obvious, especially because each phase of the AP (described in Sect. 2) has its own time and space scales. Any choice made for $T$ and $L$ in a dimensional scaling specific to a phase is justified by the fact that it is preferable to have a maximal value for $D_{\tilde{t}} \widetilde{u}$ and $D_{\widetilde{x}} \widetilde{u}$ of about 1 during this phase. This way, it is easier to compare the contribution of every term in the differential equations during this phase.

In the following, we propose time and space scales based on what is observed for a healthy human heart. The parameters defined for three different dimensional scalings and the parameters of the model are given in Table 2. A typical AP upstroke (depolarisation) of a ventricle is used to scale the time and space for the first proposed dimensional scaling Adim1. The excited phase duration and width are used to scale for the second dimensional scaling Adim2. The last dimensional scaling Adim3 is not justified by any physiological manifestation but is practical in terms of units (time measured in $m s$ and space in $\mathrm{mm}$ ).

\subsection{A nondimensionalisation specific to the asymptotic analysis}

The estimation of the magnitude of all terms in model (3.2)-(3.8) is crucial to perform the asymptotic analysis of Section 4. To achieve this comparison for the MS model, the time scales $T_{f}$ and $T_{g}$, associated respectively with source terms $f$ and $g$, are introduced. The resulting time parameters are not only dimensionless, but 
of order one as they are rescaled with $\tau_{\text {in,out }}=\widetilde{\tau}_{\text {in,out }} T_{f}$ and $\tau_{\text {open,close }}=\widetilde{\tau}_{\text {in,out }} T_{g}$. Note that this method is general enough so that it is applicable for any ionic model constructed with source terms of the form (3.1). From the equations (2.6)-(2.7), $T_{f}$ (resp. $T_{g}$ ) depends only on $\tau_{\text {in }}$ and $\tau_{\text {out }}$ (resp. $\tau_{\text {open }}$ and $\tau_{\text {close }}$ ). From the values proposed for the parameters $\tau$ 's, $T_{f}$ is necessarily a short time scale and $T_{g}$ is a long time scale. The following nondimensionalisation, using these two new time scales, is brought in order to facilitate the asymptotic analysis.

$$
\begin{array}{rr}
\frac{\partial v}{\partial \widetilde{t}}=\frac{T}{T_{g}} \widetilde{g}(\widetilde{u}, v) & \text { in } H \\
\frac{\partial \widetilde{u}}{\partial \widetilde{t}}+\frac{T}{T_{f}} \widetilde{f}(\widetilde{u}, v)=N \widetilde{\nabla} \cdot\left(\widetilde{\sigma_{i}} \widetilde{\nabla}\left(\widetilde{u}+\widetilde{u_{e}}\right)\right) & \text { in } H
\end{array}
$$

Remark that when numerical simulations are performed with nondimensional equations, it is neither useful nor practical to find values for the time scales $T_{f}$ and $T_{g}$. Absorbing $T / T_{f}$ (resp. $T / T_{g}$ ) in $\widetilde{f}$ (resp. in $\widetilde{g}$ ) is a better choice in this circumstance, where the only relevant nondimensional number is $N$ and the only parameters that remain to be fixed are $T$ and $L$. This is why there is a factor $T / T_{f}\left(\operatorname{resp} . T / T_{g}\right)$ between the $\widetilde{f}(\operatorname{resp}$. in $\widetilde{g}$ ) of equations (3.9)-(3.10) and equations (3.2)-(3.3).

\section{Asymptotic analysis}

This section is devoted to the analysis of solution features via an asymptotic analysis. The choice of phenomenological models with the minimal number of variables to describe the ionic activity offers tremendous advantage here, as their 0D (resp. 1D) solutions can be readily analysed in the $(u, v)$ phase space (resp. $\left(u_{x}, u, v\right)$ phase space). This is done for instance in [5] and [16] for the FHN equations using singular perturbation theory. Britton [5] uses the smallness of a parameter $\epsilon$, which is related to a ratio of time and space scales but is not connected with physical parameters such as conductivity $\sigma$, cell surface-to-volume ratio $\chi$, membrane specific capacitance $C_{\mathrm{m}}$. They even prove the convergence in terms of the parameter $\epsilon$ of the numerical solutions with diffusion to the asymptotic solution.

Our approach is different because the equations are all set with their physiological parameters (see Sect. 3). The smallness of some terms compared to others allows to simplify the equations and get the asymptotic 1D model and solutions. There is no convergence result because varying the parameters is not an option. The problem is addressed in this way in the hope of finding as many relations as possible between the parameters of the equations and the features of the solution.

This is also why the MS model is chosen. There already exist many results in the literature allowing to characterise the solution of the MS model in terms of the model parameters. In [11], Mitchell and Schaeffer first characterised the $0 \mathrm{D}$ solution by giving mappings predicting for instance the next APD according to the last APD, diastolic interval and pacing interval. Schaeffer and et al. [29,30] refined the MS model and the associated prediction mappings. Starting from these valuable results, we push the analysis further which leads to the main achievement of this paper: being able to control locally (in space) a propagated AP by using a systematic way of setting the model parameters (see Sect. 5).

\subsection{Definitions}

We define the following time scales to quantify all phases of the AP. During the depolarisation (phase I), the AP can be characterised with its upstroke duration $T_{\mathrm{up}} . T_{\mathrm{AP}}$ is defined to measure the duration of the excitation phase (phase II) and it is commonly called the AP duration (APD) in the literature. Phase III is measured with the downstroke duration $T_{\text {down }}$, defined to characterise the repolarisation duration. Finally, the recovery duration $T_{\text {rec }}$ characterises the phase IV which corresponds to a part of the refractory period, or could be also viewed as the diastolic interval depending on the value of the recovery threshold. This will be clarified in Section 4.4.2. 
Every temporal manifestation occurring in the AP is propagated in the tissue. The propagation of the AP in a tissue due to the conductive properties of the media adds a spatial dimension to the phenomenon. Hence the AP has to be quantified in terms of space scales considering the propagation at a given speed $c$. For the overall description of the propagated AP, we define also the upstroke length $L_{\text {up }}$, the excited phase length $L_{\mathrm{AP}}$, the downstroke length $L_{\text {down }}$ and the recovery length $L_{\text {rec }}$. These spatial scales are going to be used in the dimensional analysis in order to compare the magnitude of phenomena, as well as in the asymptotic analysis in order to allow a spatial description of the propagated AP.

Remark 4.1. The depolarisation can be characterised also with the maximum rate of depolarisation of the cells $\mathrm{d} V / \mathrm{d} t_{\max }$. This way of measuring the depolarisation is not going to be used.

The time of depolarisation (resp. repolarisation), which is the time taken for the depolarisation (resp. repolarisation) front to sweep the heart, is commonly used to describe the dynamic associated with the propagation. These values can be recovered with the speed of propagation and the size of the heart.

\subsection{Analysis of the phase space}

We start analysing the 0D system in order to understand the behaviour of an isolated cardiac cell. For the MS model, as for any 2 variable models, the work relies on a phase plane analysis. The nullclines and the equilibrium points are defined below and connected with the four phases of the AP.

First of all, when there is no propagation involved, the nondimensional equations (3.2)-(3.8) reduce to the simple system

$$
\begin{aligned}
\frac{\partial \widetilde{u}}{\partial \widetilde{t}}+\frac{T}{T_{f}} \widetilde{f}(\widetilde{u}, v) & =0, \\
\frac{\partial v}{\partial \widetilde{t}} & =\frac{T}{T_{g}} \widetilde{g}(\widetilde{u}, v) .
\end{aligned}
$$

The modified MS model retained is endowed with the following nondimensional source terms:

$$
\begin{gathered}
\tilde{f}(\widetilde{u}, v)=\frac{1}{\widetilde{\tau}_{\text {in }}} v(\widetilde{u}+a)^{2}(\widetilde{u}-1)+\frac{1}{\widetilde{\tau}_{\text {out }}} \widetilde{u} \\
\widetilde{g}(\widetilde{u}, v)= \begin{cases}\frac{1}{\widetilde{\tau}_{\text {open }}}(1-v) & \text { for } \widetilde{u}<\widetilde{u}_{\text {gate }}, \\
-\frac{1}{\widetilde{\tau}_{\text {close }}} v & \text { for } \widetilde{u} \geq \widetilde{u}_{\text {gate }}\end{cases}
\end{gathered}
$$

where the time scale $\widetilde{\tau}$ are nondimensionalised with $T_{f}$ and $T_{g}$, and $\tau_{\text {close }}$ equals $\tau_{\text {close }}(\widetilde{u})$ of equation $(2.9)$. In the following the $\sim$ are removed for the sake of convenience. Remark that the source terms given in $[11,29,30]$, referring to $f(u, v)$ of equation (2.8) and $g(u, v)$ of equation (2.7), are partially nondimensionalised. On the one hand, the time scales are dimensional (e.g. $\tau_{\text {in }}=0.3 \mathrm{~ms}, \tau_{\text {out }}=6 \mathrm{~ms}, \tau_{\text {open }}=130 \mathrm{~ms}$, etc.). On the other hand, $u$ and $v$ are nondimensional (e.g. $u_{\text {gate }}=0.13$ ) because they both vary within the interval $[0,1]$ given standard initial conditions $(u(0), v(0)) \in(0,1) \times(0,1)$.

In the MS model, the transmembrane potential $u$ is considered as the fast variable and the recovery variable $v$ is considered as the slow variable. The solutions of the original MS model and modified MS models are illustrated in Figure 2. Their respective phase spaces are illustrated with the four phases in Figure 3. The phase spaces are going to be thoroughly described for a better understanding of the asymptotic analysis.

Remark 4.2. The analysis of the modified version of the MS model featuring the overshoot is very similar to that of the original MS model when $u_{\text {gate }}<u_{\text {sldn }}$; the nullclines are the same though the vector field is different in the half-plane $u>u_{\text {sldn }}$. 


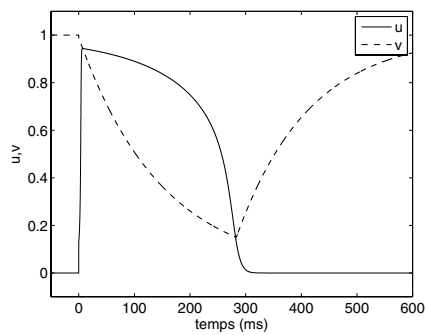

(a) Original MS model $(a=0$ and $\left.\tau_{\text {close }}=\tau_{\text {sclose }}\right)$.

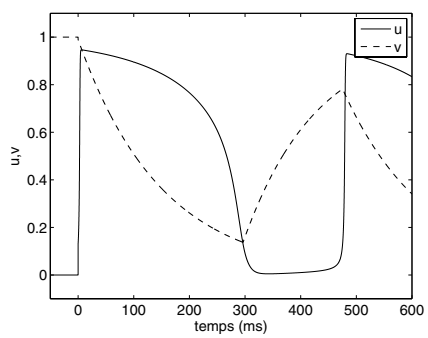

(b) Modified MS model illustrating an auto-excitable behaviour $\left(a=0.02\right.$ and $\left.\tau_{\text {close }}=\tau_{\text {sclose }}\right)$.

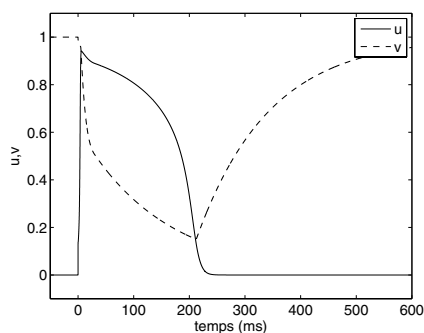

(c) Modified MS model illustrating the overshoot after the depolarisation (with nondimensional parameters $a=0, u_{\text {sldn }}=0.89, \tau_{\text {sclose }}=150$, $\tau_{\text {fclose }}=10$ and $\tau_{\text {close }}(u)$ given by equation (2.9)).

FiguRE 2. Numerical simulation of the electrical activity of a single cell using various versions of the MS model. With the characteristic time $T=1 \mathrm{~ms}$, all simulations use the nondimensional parameters $\tau_{\text {in }}=0.3, \tau_{\text {out }}=6, \tau_{\text {open }}=130$ and $u_{\text {gate }}=0.13$.

For the original MS model (see Fig. 3a), there is a local minimum on the nullcline $f(u, v)=0$ at $\left(u_{*}, v_{*}\right)=$ $\left(1 / 2,4 \tau_{\text {in }} / \tau_{\text {out }}\right)$. Note that the point at which this minimum occurs is not on the solution curve $(u(v), v)$. This local minimum separates the nullcline in 2 branches: the left-hand side branch is denoted $u=h_{2}(v)$ and the right-hand side branch $u=h_{3}(v)$ with

$$
u=h_{2,3}(v)=\frac{1}{2} \mp \frac{1}{2} \sqrt{1-4 \frac{\tau_{\text {in }}}{\tau_{\text {out }} v}} .
$$

There are 3 equilibrium points, $(0,1),\left(h_{2}(1), 1\right)$ and one on the line $u=u_{\text {gate, }}$ precisely the point $\left(u_{\text {gate }}, \tau_{\text {in }} /\left[\tau_{\text {out }} u_{\text {gate }}\left(1-u_{\text {gate }}\right)\right]\right)$. For the set of parameters given above, the equilibria $(0,1)$ and $\left(h_{2}(1), 1\right)$ are saddle points. Their stability can be studied with a standard analysis of the linearised version of system (4.1)-(4.2). The third equilibrium $\left(u_{\text {gate }}, h_{2}(v)\right)$ is an unstable focus, which can be seen by inspection of the vector field in the phase plane. During phase I the solution starts at the point $A$, corresponding to initial condition, then quickly reach the point $B$. From point $A$ to $B$, the recovery variable $v$ barely varies. Phase II is characterised by a solution following very closely the branch $u=h_{3}(v)$ of the nullcline $f(u, v)=0$, i.e. from point $B$ to $C$. During phase III, the solution leaves the nullcline $f(u, v)=0$ at the point $C, u$ goes fast to zero while $v$ varies only slightly. In phase IV the solution goes along the nullcline $u=0$ from the point $D$ while $v$ increases slowly. The solution reaches asymptotically the equilibrium point $(0,1)$.

For the modified MS model (see Fig. 3b), the nullcline $f(u, v)=0$ have a local minimum at $\left(u_{*}, v_{*}\right)$ with $u_{*}=1 / 4(1-\sqrt{1-8 a})$ and a local maximum at $\left(u^{*}, v^{*}\right)$ with $u^{*}=1 / 4(1+\sqrt{1-8 a})$. These local extrema separate the nullcline in 3 branches denoted by $u=h_{1}(v), u=h_{2}(v)$ and $u=h_{3}(v)$ with $h_{1}(v) \leqslant h_{2}(v) \leqslant h_{3}(v)$. The solution starts at the initial point $A$ and follows a trajectory close to that of the original MS model until phase IV, where the solution goes along $u=h_{1}(v) \equiv 0$ from the point $D$ while $v$ increases slowly. The solution eventually leaves the nullcline near the point $\left(u^{*}, v^{*}\right)$. Finally, the solution goes again by itself in the phase I (from point $A^{\prime}$ ) after a complete cycle. This model is clearly suitable for auto-excitable cells because of the repeating cycles. There is no assumption here about the periodicity of the solution.

An asymptotic analysis could be done by regarding the 0D solution separately in its phases I, II, III and IV. In both models phases I and III are characterised by a time scale that is so short for the slow variable $v$ that it remains almost constant during these periods. The solution moves along the nullclines during phases II and IV. 


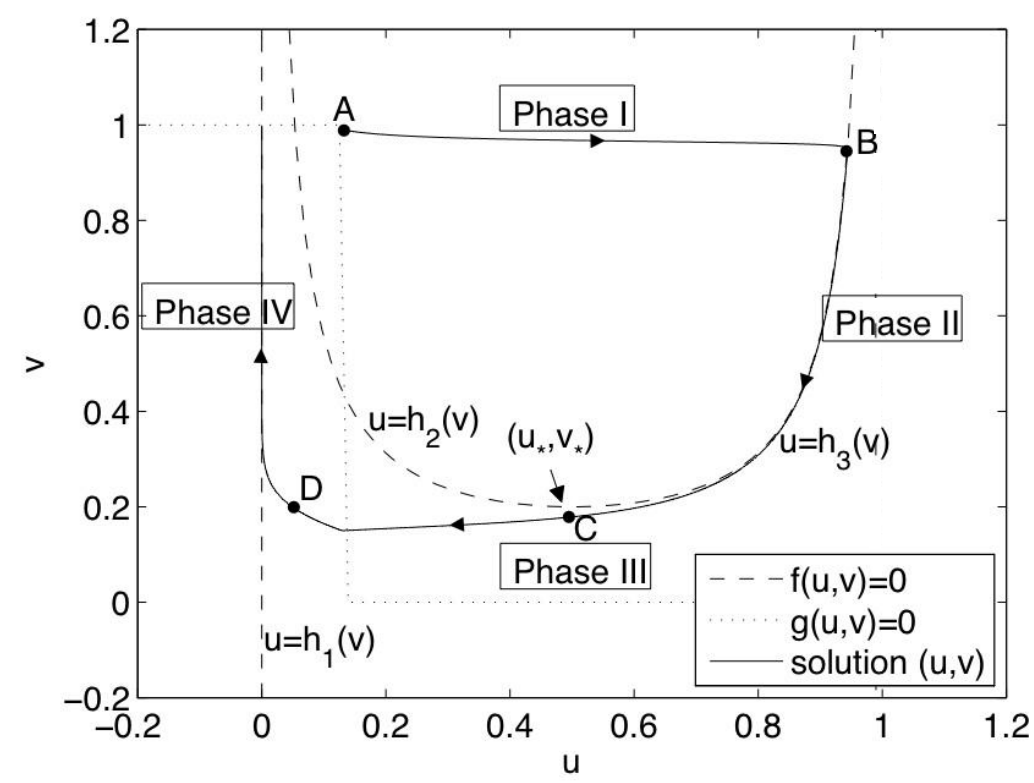

(a) The phase plane for 0D original MS model with a numerical solution.

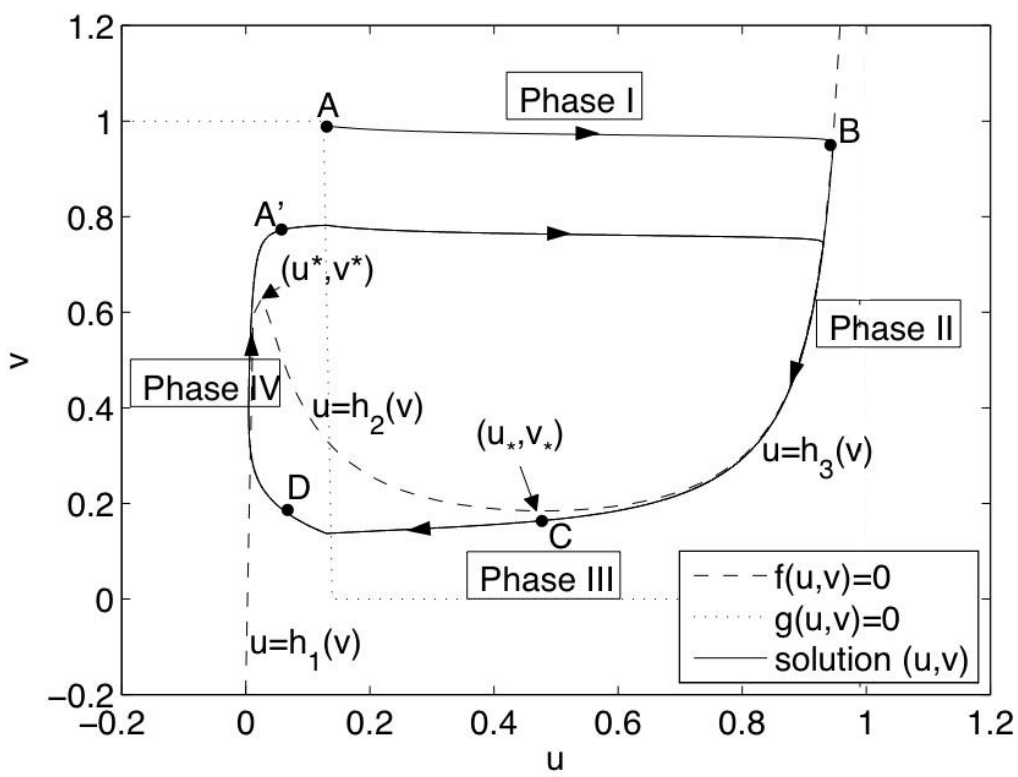

(b) The phase plane for 0D modified MS model with a numerical solution for $a=0.02$.

Figure 3. The phase space for both the MS and modified MS models. Numerical simulations are performed with $T=0.001 \mathrm{~s}, \tau_{\text {in }}=0.3(\mathrm{~ms}), \tau_{\text {out }}=6(\mathrm{~ms}), \tau_{\text {open }}=130(\mathrm{~ms}), \tau_{\text {close }}=150$ (ms) and $u_{\text {gate }}=0.13$. See text for definitions of curves, points, etc identified. 
Each part of the solution can be approximated with a simplified trajectory (moving along a nullcline or at a constant $v$ ). The 0D asymptotic solution is defined by connecting these simplified trajectories together.

We are rather interested in an asymptotic analysis of the 1D solution. For this purpose we explore the phenomenological MS model coupled with a 1D model of propagation. Using the nondimensional 1D bidomain model ( $\sim$ are removed for simplicity) with a constant conductivity, the problem consists in finding $\left(u, u_{e}, v\right)$ over the spatial domain $\left[0, x_{\max }\right]$ such that

$$
\begin{aligned}
\frac{\partial u}{\partial t}+\frac{T}{T_{f}} f(u, v) & =N_{i} \frac{\partial^{2}\left(u+u_{e}\right)}{\partial x^{2}}, \\
\sigma_{i} \frac{\partial^{2} u}{\partial x^{2}}+\left(\sigma_{i}+\sigma_{e}\right) \frac{\partial^{2} u_{e}}{\partial x^{2}} & =0 \\
\frac{\partial v}{\partial t} & =\frac{T}{T_{g}} g(u, v, t),
\end{aligned}
$$

with $N_{i}=T \sigma_{i} / C_{\mathrm{m}} \chi L^{2}$ the nondimensional number associated to the intracellular medium of conductivity $\sigma_{i}$. On the boundaries $x=0$ and $x=x_{\max }$, homogeneous Neumann boundary conditions are applied for both $u$ and $u_{e}$. A zero mean condition can be applied on the potential $u_{e}$ so that the degeneracy is removed.

The following monodomain model is equivalent to the bidomain model in 1D: Find $(u, v)$ over $\left[0, x_{\max }\right]$ such that

$$
\begin{aligned}
\frac{\partial u}{\partial t}+\frac{T}{T_{f}} f(u, v) & =N_{\text {harm }} \frac{\partial^{2} u}{\partial x^{2}}, \\
\frac{\partial v}{\partial t} & =\frac{T}{T_{g}} g(u, v, t),
\end{aligned}
$$

with $N_{\text {harm }}=T \sigma_{\text {harm }} / C_{\mathrm{m}} \chi L^{2}$ and $\sigma_{\text {harm }}=\left(\sigma_{i}^{-1}+\sigma_{e}^{-1}\right)^{-1}$ is the harmonic average of the intra and extracellular conductivities. The two models being equivalent, they give exactly the same solutions with the main advantage that the monodomain model will be less computationally intensive in numerical tests performed to verify our asymptotic solution.

The next step is to verify that under physiologically plausible conditions, the MS model with spatial propagation is suitable for an asymptotic analysis. A 1D solution is computed beforehand with the parameters of the third column of Table 2 together with the dimensioning parameters $L=0.001 \mathrm{~m}, T=0.001 \mathrm{~s}$ and $N_{\text {harm }}=0.05$. The domain is the interval $[0,600]$ and the simulation is performed over the time interval $[0,1000]$. The initial condition is given by

$$
u(0)= \begin{cases}0 & \text { for } x \in[0,570) \\ 0.8 & \text { otherwise }\end{cases}
$$

and $v(0)=0.99$.

At one coordinate point $(x=300)$, the solution is plotted in the $(u, v)$-phase plane, with its corresponding nullclines (see Fig. 4). This figure shows obvious similarities between the solution of the system with diffusion in $1 \mathrm{D}$ and the solution of the equations for an isolated cell in $0 \mathrm{D}$.

Again, it follows that the solution can be separated into four phases. These four phases are matched together to give the so-called asymptotic $1 \mathrm{D}$ solution.

The time and space scales of the asymptotic solution are defined as it was done in Section 4.1 for the scales $T_{\text {up }}$, $L_{\text {up }}$, etc, of the exact solution. The related scales for the asymptotic solution are denoted with a hat $(\wedge)$. For instance, the asymptotic upstroke duration is denoted by $\widehat{T}_{\text {up }}$, the asymptotic upstroke width by $\widehat{L}_{\text {up }}$, etc. In practice, scales of the exact solution can only be obtained through accurate numerical computations and for a limited set of parameter values, while algebraic expressions will be obtained for all asymptotic scales. 


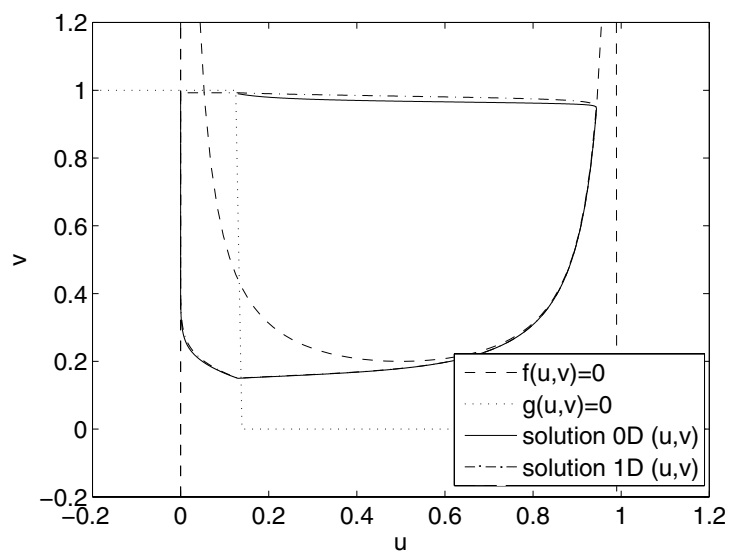

Figure 4. 0D and 1D solutions plotted in the phase space $(u, v)$ of the $0 \mathrm{D}$ system.

\subsection{Asymptotic characterisation of the depolarisation front}

In order to find the asymptotic regime of the depolarisation, the magnitude of each term in equations (4.4)-(4.5) is estimated during phase I. The leading terms will form the equations to solve for defining the asymptotic solution during this phase. Known values for the depolarisation duration and width can be used (see Tab. 2) for this purpose. For instance, the ventricle characteristic depolarisation duration is about $1 \mathrm{~ms}$ and the speed of the wave is about $0.5 \mathrm{~m} / \mathrm{s}$. Using $T=1 \mathrm{~ms}$ and $L=0.5 \mathrm{~mm}$, the terms in the equations are of order one and they are weighted by the dimensionless numbers multiplying them. Because $T / T_{f} \sim 1$, $N_{\text {harm }}=N_{\text {up }}=0.2$ and $T / T_{g} \sim 7 \times 10^{-3}$, the term $T / T_{g} g(u, v)$ is negligible compared to the other terms. The asymptotic solution in phase I is given by $(u, v)$ over $\left[0, x_{\max }\right] \times\left[0, t_{\max }\right]$ such that

$$
\begin{array}{r}
\frac{\partial u}{\partial t}+\frac{T}{T_{f}} f\left(u, v_{-}\right)=N_{\text {up }} \frac{\partial^{2} u}{\partial x^{2}} \text { with } u(t=0)=u_{0}, \\
\frac{\partial v}{\partial t}=0 \Leftrightarrow v=v(t=0)=v_{-},
\end{array}
$$

with the initial conditions given in Section 4.2. The value of $v$ at which phase I occurs will be called $v_{-}$, where $v_{-}$can be interpreted as the value of $v$ facing the arrival of the wave. As the wave can be paced at almost any value of $v, v_{-}$is not specified unless an isolated wave is analysed. In this case, $v_{-}=1$, i.e. the value at equilibrium.

We can adjust the parameters $T$ and $L$ in this asymptotic model for phase I so that the asymptotic upstroke has a duration of 1 and a width of 1 in the nondimensional frame, which amounts to set $T=\widehat{T}_{\text {up }}$ and $L=\widehat{L}_{\text {up }}$. The system then reduces to a single equation

$$
\frac{\partial u}{\partial t}+\frac{\widehat{T}_{\mathrm{up}}}{T_{f}} f\left(u, v_{-}\right)=N_{\mathrm{up}} \frac{\partial^{2} u}{\partial x^{2}}
$$

where the solution $u$ goes from $u(0)$ to $h_{3}\left(v_{-}\right)$.

It is known that under appropriate assumptions, reaction-diffusion equations such as equation (4.8) have travelling wave solutions, i.e. solutions of the form $u(x, t)=\nu(x+c t)$, propagating at the speed $c$ in the direction of decreasing $x$ if $c>0$. As a simple and well-known example, for $f\left(u, v_{-}\right)=-\alpha u$, the existence of a planar travelling wave solution can be easily proved in a constructive way (see [5]). This wave is asymptotically stable, with wave speeds approaching the constant value $c$ as $t \rightarrow \infty$ for any initial solution that leads to the 
asymptotic state. However, for more complicated ionic models, the proof of existence of travelling waves requires care as it is highly dependent on the form of the ionic terms. For instance, the 1D travelling solution of the FHN model is thoroughly analysed in $[9,14]$ including the existence of different modes. For the MS model, it is shown in [2] that unidimensional travelling pulse solutions exist.

We summarise this argument and clarify notations here for the sequel of the text. The necessary conditions for the existence of travelling wave can be studied by analysing the $\left(\nu, \nu^{\prime}\right)$-phase plane. The speed $c$ must be properly set, say to $c^{*}$, so that a travelling wave exists, which amounts to the existence of a heteroclinic connection of the equilibrium points $\left(\nu, \nu^{\prime}\right)=(0,0)$ and $\left(\nu, \nu^{\prime}\right)=\left(h_{3}\left(v_{-}\right), 0\right)$. It is easy to show that non-monotonous orbits starting at $\nu=0$ and reaching $\nu=h_{3}\left(v_{-}\right)$are impossible. By monotonicity, the heteroclinic orbit is attained in a unique way referring to a specific speed $c^{*}$, where the uniqueness holds for any fixed value of $v_{-}$. If a bounded positive speed for the monotonous orbit can be found, the necessary conditions for the existence of such heteroclinic orbit are going to be all satisfied. The next step concerns the computation of that unique and bounded speed.

\subsubsection{Matching condition for the asymptotic travelling wave solution}

Using similar arguments as in [5], the propagation speed will be found by analysing the formation or the "root" of the travelling wave in 1D. The travelling wave is formed during phase I, so the speed is that of the depolarisation front. Remark that $c=\widehat{L}_{\text {up }} / \widehat{T}_{\text {up }}$ and dimensionless speed is equal to 1 .

The asymptotic travelling wave $u(x, t)=\nu(x+c t)=\nu(s)$ solves the equation (4.8), which gives

$$
c \nu^{\prime}+\frac{T}{T_{f}} f\left(\nu, v_{-}\right)=N \nu^{\prime \prime} .
$$

A matching condition for the speed $c=c^{*}$ is obtained by multiplying (4.9) by $\nu^{\prime}$ and integrating along the heteroclinic orbit that connects the points $\nu=0$ and $\nu=h_{3}\left(v_{-}\right)$as $s \rightarrow \mp \infty$ :

$$
c^{*} \int_{-\infty}^{+\infty} \nu^{\prime}(s)^{2} \mathrm{~d} s=-\frac{T}{T_{f}} \int_{0}^{h_{3}\left(v_{-}\right)} f\left(\nu, v_{-}\right) \mathrm{d} \nu .
$$

The dimensional asymptotic speed $\widehat{c}$ is then

$$
\widehat{c}=\frac{L}{T} c^{*}=-L \frac{F_{\mathrm{AB}}\left(v_{-}\right)}{\int_{-\infty}^{+\infty} \nu^{\prime}(s)^{2} \mathrm{~d} s}
$$

with

$$
\begin{aligned}
F_{\mathrm{AB}}\left(v_{-}\right) & \equiv \frac{1}{T_{f}} \int_{0}^{h_{3}\left(v_{-}\right)} f\left(\nu, v_{-}\right) \mathrm{d} \nu \\
& =\left[\frac{v_{-}}{\tau_{\text {in }}}\left(\frac{h_{3}\left(v_{-}\right)^{4}}{4}-\frac{h_{3}\left(v_{-}\right)^{3}}{3}\right)+\frac{1}{2} \frac{h_{3}\left(v_{-}\right)^{2}}{\tau_{\text {out }}}\right] .
\end{aligned}
$$

For $v_{-} \in\left(4 \tau_{\text {in }} / \tau_{\text {out }}, 1\right], F_{\mathrm{AB}}\left(v_{-}\right)$(in $s^{-1}$; recall that the $\tau^{\text {'s }}$ are nondimensionalised with $\left.T_{f}\right)$ is well defined and negative as $f\left(\nu, v_{-}\right)<0$ during phase I.

As $L=\widehat{L}_{\text {up }}$ and $T=\widehat{T}_{\text {up }}$ are used to nondimensionalise the equations in this section, the dimensionless speed $c^{*}$ is equal to 1 . A consequence is that the dimensional asymptotic upstroke duration can be written as

$$
\widehat{T}_{\mathrm{up}}=-\frac{1}{F_{\mathrm{AB}}\left(v_{-}\right)} \int_{-\infty}^{+\infty} \nu^{\prime}(s)^{2} \mathrm{~d} s .
$$

The expression (4.10) for the asymptotic speed is a known result derived for instance in [16] while analysing the FHN model. It cannot be used directly to predict the speed of the travelling wave because the value of the integral $\int_{-\infty}^{+\infty} \nu^{\prime}(s)^{2} \mathrm{~d} s$ is not known so far. With the analysis of Sections 4.3.2 and 4.4.1, this integral will be estimated in terms of the parameters of the equations. 


\subsubsection{Estimation of the speed using an ansatz}

In this section, an approximation of the travelling wave solution is constructed for the phase I. At the very beginning of the formation of the wave, the rise of the potential is assumed to occur exponentially. The ansatz then reads as

$$
\nu(s)=u_{\text {gate }} e^{s / \delta},
$$

where $\delta$ is dimensionless and is chosen to be

$$
\delta=\frac{1}{\ln \left(\frac{h_{3}\left(v_{-}\right)}{u_{\text {gate }}}\right)}
$$

for the upstroke to be between $u_{\text {gate }}$ and $h_{3}\left(v_{-}\right)$and have a width of 1 in the nondimensional frame (because nondimensionalisation is based on $L=\widehat{L}_{\mathrm{up}}$ and $T=\widehat{T}_{\mathrm{up}}$ ).

In the same spirit as collocation methods, we want the ansatz (4.12) to solve the equation (4.9) at a given point, say at the location where $\nu=u_{\text {gate }}$. Other collocation points can be chosen to end up with similar results. The choice of the point for which $\nu=u_{\text {gate }}$ is the most appropriate since this is where the solution resembles the most an exponential. Substituting $e^{s / \delta}$ in equation (4.9) gives

$$
\frac{c^{* *}}{\delta}+\psi\left(u_{\text {gate }}, v_{-}\right)=\frac{N}{\delta^{2}}
$$

at $s=0$, where

$$
\psi\left(u_{\text {gate }}, v_{-}\right)=\frac{1}{\tau_{\text {in }}} v_{-} u_{\text {gate }}\left(u_{\text {gate }}-1\right)+\frac{1}{\tau_{\text {out }}}
$$

and $c^{* *}$ is the dimensionless speed of a travelling wave of this shape. As $L=\widehat{L}_{\text {up }}$ and $T=\widehat{T}_{\text {up }}$, the dimensionless speed $c^{* *}$ is again equal to 1 , then

$$
\frac{\delta^{2}}{\widehat{T}_{\mathrm{up}}} \psi\left(u_{\text {gate }}, v_{-}\right)+\frac{\delta}{\widehat{T}_{\mathrm{up}}}=\frac{\sigma}{C_{\mathrm{m}} \chi \widehat{L}_{\mathrm{up}}^{2}} .
$$

Equations (4.11) and (4.13) provide relations between $\widehat{T}_{\text {up }}$, the ionic model parameters (either through the functions $F_{\mathrm{AB}}$ or $\psi$ ) and the diffusion constant $\sigma /\left(C_{\mathrm{m}} \chi\right)$ parameterising the conduction effects. We assume that the depolarisation time is the same for an isolated myocyte as for coupled cells within the cardiac tissue. Basically, if a cell is excited by an external stimulus, the depolarisation is controlled by ionic channel opening and closing while the conduction properties of the surrounding media has little if any impact on the channel activity. This assumption is confirmed with the MS model: there is a very slight difference between the transmembrane potential evolution of an isolated cell and a cell surrounded by other cells. We do not claim that this remains true for other models, e.g. which take into account of the load imposed by connected cells. This implies that $T_{\text {up }}$ and its approximation $\widehat{T}_{\mathrm{up}}$ must be independent from the diffusion constant $\sigma /\left(C_{\mathrm{m}} \chi\right)$, fact which is confirmed with numerical solutions as shown in Figure 5. From (4.11), one deduces that the nondimensional integral $\int_{-\infty}^{+\infty} \nu^{\prime}(s)^{2} \mathrm{~d} s$ does not depend on the diffusion constant $\sigma /\left(C_{\mathrm{m}} \chi\right)$. Similarly, the left-hand-side of (4.13) being constant for given values of $\tau$ 's, $u_{\text {gate }}$ and $v_{-}$, the depolarisation length $\widehat{L}_{\text {up }}$ behaves as $\sqrt{\sigma /\left(C_{\mathrm{m}} \chi\right)}$, and as $\widehat{c}=\widehat{L}_{\text {up }} / \widehat{T}_{\text {up }}, \widehat{c}$ behaves as $\sqrt{\sigma /\left(C_{\mathrm{m} \chi} \chi\right)}$ too. The asymptotic behaviours of the travelling wave speed and upstroke width can be verified numerically as shown in Figure 6.

In summary, the characterisation of the depolarisation front raises the following important facts, for given values of $\tau$ 's, $u_{\text {gate }}$ and $v_{-}$.

1. The asymptotic upstroke duration $\widehat{T}_{\mathrm{up}}$ is independent from the diffusion constant $\sigma /\left(C_{\mathrm{m}} \chi\right)$.

2. The asymptotic upstroke width $\widehat{L}_{\text {up }}$ behaves as $\sqrt{\sigma /\left(C_{\mathrm{m}} \chi\right)}$. This holds also for the numerical solution.

3. The asymptotic speed $\widehat{c}$ behaves as $\sqrt{\sigma /\left(C_{\mathrm{m}} \chi\right)}$. This holds also for the numerical solution.

4. The dimensionless number $\widehat{N}_{\text {up }}$ calculated using the length and time scales of the upstroke phase is constant with respect to the diffusion constant $\sigma /\left(C_{\mathrm{m}} \chi\right)$. 


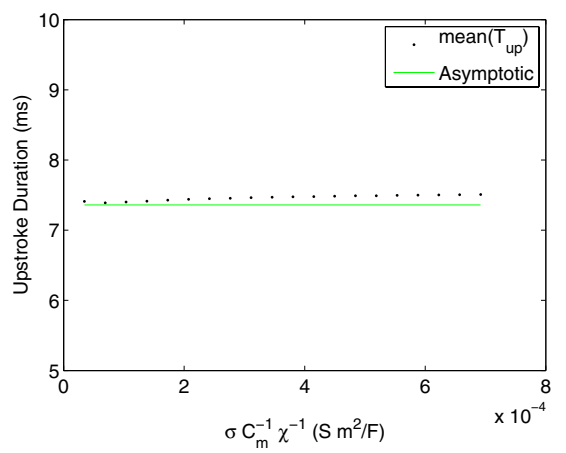

FiguRE 5. Upstroke duration versus $\frac{\sigma}{C_{\mathrm{m}} \chi}$ for the MS model. The asymptotic duration will be discussed in Section 4.5.

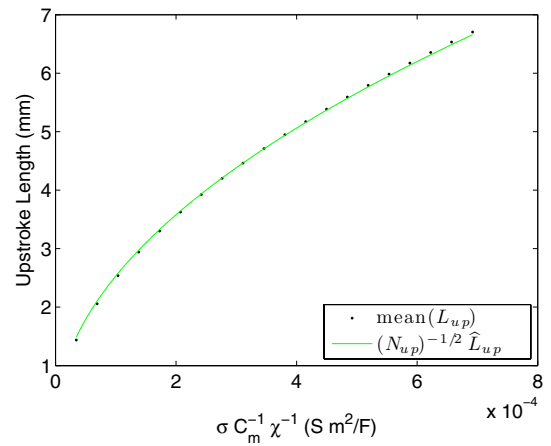

(a) Upstroke length.

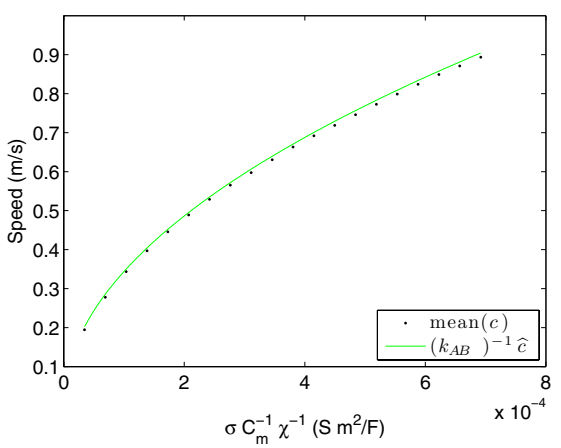

(b) Speed.

FiguRE 6. Upstroke length (a) and speed (b) versus $\frac{\sigma}{C_{\mathrm{m}} \chi}$ for the MS model. The asymptotic prediction is detailed in Section 4.5.

\subsection{Overall characterisation of the asymptotic solution}

In this section, the features of the asymptotic solution are studied. With a judicious combination of assumptions, algebraic expressions for short time scale features (durations) are derived, followed by long time scale features and finally, spatial features (length and speed). These expressions depend on the parameters of the model, and describe the asymptotic solution. In Section 4.5, these results are used to predict the numerical solution of the action potential.

\subsubsection{The asymptotic short time scales: Phases I and III}

In order to find an expression for the nondimensional asymptotic upstroke duration $\widehat{T}_{\text {up }}$, the remaining integral in equation (4.11) has to be somehow estimated. To do so, a linear rise of $u$ is assumed during the upstroke and

$$
\nu^{\prime} \simeq \begin{cases}h_{3}\left(v_{-}\right) & \text {for } 0 \leqslant s \leqslant 1 \\ 0 & \text { otherwise }\end{cases}
$$


The asymptotic upstroke duration is then deduced with simple calculation

$$
\widehat{T}_{\mathrm{up}}=-\frac{1}{F_{\mathrm{AB}}\left(v_{-}\right)} h_{3}\left(v_{-}\right)^{2} .
$$

Note that the asymptotic upstroke duration depends nonlinearly on the parameters $\tau_{\text {in }}, \tau_{\text {out }}, v_{-}$and eventually $u_{\text {gate }}$ (see Rems. 4.3 and 4.4 below). In case the action potential pulse is isolated the solution leaves and returns to the equilibrium point $(u, v)=(0,1)$ in the phase space. In this case, using $v_{-}=1$ gives a very good estimate.

Transposing this way of measuring the asymptotic upstroke duration into a way of measuring the upstroke duration for numerical solutions is inappropriate: using the depolarisation threshold $u=0$ to flag the initiation of the action potential is not practical. The following two remarks bring alternative ways of measuring and predicting the upstroke duration that are compatible with numerical computations and experiments.

Remark 4.3. Justified by the ansatz (4.12), an exponential rise of $u$ is assumed during the asymptotic upstroke and

$$
\nu^{\prime} \simeq \begin{cases}\frac{u_{\text {gate }}}{\delta} e^{s / \delta} & \text { for } 0 \leqslant s \leqslant 1 \\ 0 & \text { otherwise. }\end{cases}
$$

The upstroke needs to be measured between $u=u_{\text {gate }}($ at $s=0)$ and $h_{3}\left(v_{-}\right)$(at $\left.s=1\right)$. Consequently, the integral in $F_{\mathrm{AB}}$ can be estimated using

$$
\begin{aligned}
F_{\mathrm{AB}}\left(v_{-}\right) \equiv & \frac{1}{T_{f}} \int_{u_{\text {gate }}}^{h_{3}\left(v_{-}\right)} f\left(\nu, v_{-}\right) \mathrm{d} \nu \\
= & {\left[\frac{v_{-}}{\tau_{\text {in }}}\left(\frac{h_{3}\left(v_{-}\right)^{4}}{4}-\frac{h_{3}\left(v_{-}\right)^{3}}{3}\right)+\frac{1}{2} \frac{h_{3}\left(v_{-}\right)^{2}}{\tau_{\text {out }}}\right] } \\
& -\left[\frac{v_{-}}{\tau_{\text {in }}}\left(\frac{u_{\text {gate }}^{4}}{4}-\frac{u_{\text {gate }}^{3}}{3}\right)+\frac{1}{2} \frac{u_{\text {gate }}^{2}}{\tau_{\text {out }}}\right]
\end{aligned}
$$

and the asymptotic upstroke duration becomes

$$
\widehat{T}_{\mathrm{up}}=-\frac{u_{\text {gate }}}{2 \delta F_{\mathrm{AB}}\left(v_{-}\right)}\left(h_{3}^{2}\left(v_{-}\right)-u_{\text {gate }}^{2}\right) .
$$

Remark 4.4. If a linear rise between $u=u_{\text {gate }}$ and $h_{3}\left(v_{-}\right)$is assumed during the asymptotic upstroke,

$$
\nu^{\prime} \simeq \begin{cases}h_{3}\left(v_{-}\right)-u_{\text {gate }} & \text { for } 0 \leqslant s \leqslant 1 \\ 0 & \text { otherwise }\end{cases}
$$

and the asymptotic upstroke duration becomes

$$
\widehat{T}_{\text {up }}=-\frac{1}{F_{\mathrm{AB}}\left(v_{-}\right)}\left(h_{3}\left(v_{-}\right)-u_{\text {gate }}\right)^{2},
$$

using $F_{\mathrm{AB}}\left(v_{-}\right)$of Remark 4.3.

Remark 4.5. It is possible to show that the estimate (4.16) provides a lower bound for $\widehat{T}_{\text {up }}$ under the sole hypothesis that $\nu=\nu(s)$ varies between $u_{\text {gate }}$ and 1 over the interval $[0,1]$ without any further assumption on the profile for $\nu(s)$ (linear, exponential, etc). Using Jensen inequality and $c^{\star}=\widehat{L}_{\text {up }} / \widehat{T}_{\text {up }}=1$,

$$
\left[h_{3}\left(v_{-}\right)-u_{\text {gate }}\right]^{2}=\left[\int_{0}^{1} \nu^{\prime}(s) \mathrm{d} s\right]^{2} \leq \int_{0}^{1} \nu^{\prime}(s)^{2} \mathrm{~d} s \leq \int_{-\infty}^{+\infty} \nu^{\prime}(s)^{2} \mathrm{~d} s=-\widehat{T}_{\mathrm{up}} F_{\mathrm{AB}}\left(v_{-}\right),
$$

hence the lower bound. 


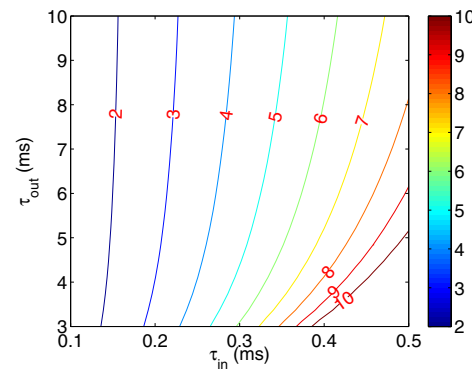

(a) Using a linear rise between 0 and $h_{3}\left(v_{-}\right)$.

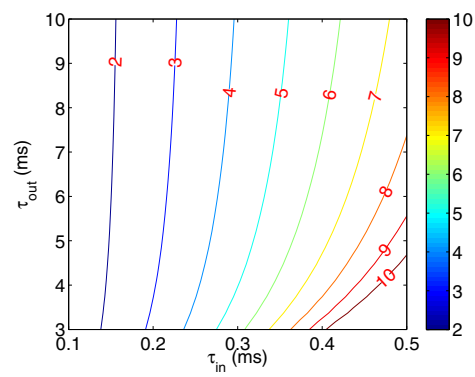

(b) Using an exponential rise between (c) Using a linear rise between $u_{\text {gate }}$ and $u_{\text {gate }}$ and $h_{3}\left(v_{-}\right)$.

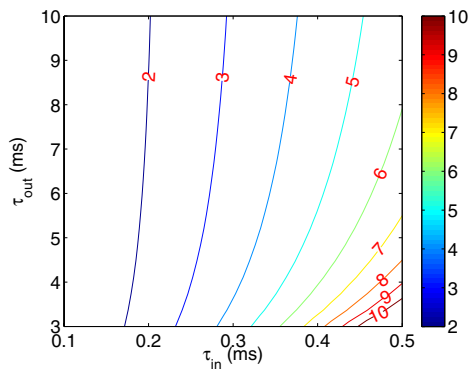

$h_{3}\left(v_{-}\right)$.

FiguRE 7. Asymptotic upstroke duration (in ms) plotted as a function of $\tau_{\text {in }}$ and $\tau_{\text {out }}$ for three approximations of the upstroke profile.

The three ways of estimating the asymptotic upstroke duration presented above give a similar behaviour whenever the parameters $\tau_{\text {in }}$ and $\tau_{\text {out }}$ are varied. This is illustrated on Figure 7 . These three asymptotic estimates of the upstroke duration being equivalent, the estimate of equation (4.15) is going to be used in the following.

For a stable travelling wave, the speed of the repolarisation front is the same as the speed of the depolarisation front, and one has $c^{*}=\widehat{L}_{\text {down }} / \widehat{T}_{\text {down }}$. In phase III, the asymptotic solution consists in a trajectory connecting $\left(u_{*}, v_{*}\right)=\left(1 / 2,4 \tau_{\text {in }} / \tau_{\text {out }}\right)$ and $\left(0, v_{*}\right)=\left(0,4 \tau_{\text {in }} / \tau_{\text {out }}\right)$. Assuming that the decay of $u$ is linear, the dimensional asymptotic downstroke duration is estimated as

$$
\widehat{T}_{\text {down }}=-\frac{u_{*}^{2}}{F_{C D}}=-\frac{1}{4 F_{C D}}
$$

with

$$
F_{C D} \equiv \frac{1}{T_{f}} \int_{1 / 2}^{0} f\left(\nu, 4 \tau_{\text {in }} / \tau_{\text {out }}\right) \mathrm{d} \nu=-\frac{1}{48 \tau_{\text {out }}} .
$$

\subsubsection{The asymptotic long time scales: Phases II and IV}

In phase II, the nondimensional equations (4.4)-(4.5) are used in order to find the asymptotic action potential duration $\widehat{T}_{\mathrm{AP}}$. Phase II being the period of excitation, the time scale $T=\widehat{T}_{\mathrm{AP}}$ would ideally be chosen. However, $\widehat{T}_{\mathrm{AP}}$ is not known yet in terms of the parameters of the equations, and $T=T_{g}$ sets a long time scale that depends only on $\tau_{\text {open }}$ and $\tau_{\text {close }}$. This is enough to compare the magnitude of the terms in the equations and estimate how good is the asymptotic approximation. Recall that $T_{f}$ depends on the parameters $\tau_{\text {in }}$ and $\tau_{\text {out }}$ of the function $f$, so it is a short time scale. With this choice of time scales, $\partial u / \partial t$ and $\partial^{2} u / \mathrm{d} x^{2}$ are of order one in magnitude, one gets $T_{g} / T_{f}=T_{\mathrm{AP}} / T_{\mathrm{up}} \sim 100$ and $\left(\sigma_{\mathrm{harm}} T\right) /\left(\chi C_{\mathrm{m}} L^{2}\right)=1 \times 10^{-3}$. It results that $T_{g} / T_{f} \gg 1 \gg\left(\sigma_{\text {harm }} T_{f}\right) /\left(\chi C_{\mathrm{m}} L^{2}\right)$ and equation (4.4) becomes $f(u, v)=0$ to leading order.

Remark 4.6. In the asymptotic analysis of the FHN model done in [16], $T_{f} / T_{g}$ can be compared to the parameter $\epsilon$ and $\left(\sigma_{\text {harm }} T_{f}\right) /\left(\chi C_{\mathrm{m}} L^{2}\right)<\epsilon^{2}$.

We assume that the asymptotic solution goes along the nullcline $f(u, v)=0$ during phase II. Matching the solution with that of phase I, the evolution of the recovery variable during phase II is described using

$$
\frac{\partial v}{\partial t}=\frac{T}{T_{g}} g\left(h_{3}(v), v\right) \text { with } v(0)=v_{-},
$$


where $v_{-}$is the value of $v$ facing the arrival of the wave $\left(v_{-}=1\right.$ for an isolated wave). The trajectory goes along the branch $u=h_{3}(v)$ and $v$ decreases until it reaches $v_{*}$ beyond which $h_{3}(v)$ ceases to exist. The dimensional time taken for this phase is

$$
\widehat{T}_{\mathrm{AP}}=T_{g} \int_{v_{-}}^{v_{*}} \frac{1}{g\left(h_{3}(v), v\right)} \mathrm{d} v
$$

where $g\left(h_{3}(v), v\right)$ is defined with dimensionless parameters and variables.

During this phase $u_{\text {gate }}<u_{*}=1 / 2$ and then $g\left(h_{3}(v), v\right) / T_{g}=-v / \tau_{\text {close }}$ so

$$
\widehat{T}_{\mathrm{AP}}=-\int_{v_{-}}^{4} \frac{\tau_{\text {in }}}{\tau_{\text {out }}} \frac{\tau_{\text {close }}}{v} \mathrm{~d} v=\tau_{\text {close }} \ln \left(\frac{\tau_{\text {out }} v_{-}}{4 \tau_{\text {in }}}\right)
$$

This is a well established result first published in [22]. With the dimensional values of $\tau$ given in Section 2.2 and $v_{-}=1$, one gets $\widehat{T}_{\mathrm{AP}} \approx 241 \mathrm{~ms}$ for the MS model $(2.6)-(2.7)$.

Remark 4.7 (Characterisation of the overshoot). The overshoot duration and height can be characterised as for the AP duration. The overshoot is a part of the phase II because the trajectory is along the branch $u=h_{3}(v)$ even if the time scale is temporarily shortened with $\tau_{\text {close }}(u)$. The overshoot features a peak of height given by $h_{3}\left(v_{-}\right)-u_{\text {sldn }}$ and duration given by the following integral

$$
\widehat{T}_{\text {peak }}=T_{g} \int_{h_{3}\left(v_{-}\right)}^{u_{\text {sldn }}} \frac{1}{g\left(u, h_{3}^{-1}(u)\right)} \frac{\mathrm{d} v}{\mathrm{~d} u} \mathrm{~d} u
$$

with $v=h_{3}^{-1}(u)=\tau_{\text {in }} /\left[\tau_{\text {out }} u(1-u)\right]$. As $1 / \tau_{\text {close }}(u)=m(u+b)$ with $m=\left(1 / \tau_{\text {fclose }}-1 / \tau_{\text {sclose }}\right) /\left(1-u_{\text {sldn }}\right)$ and $b=\left(1-u_{\text {sldn }}\right) /\left(1-\tau_{\text {fclose }} / \tau_{\text {sclose }}\right)-1$ the integral becomes

$$
=-\frac{1}{m} \int_{h_{3}\left(v_{-}\right)}^{u_{\text {sldn }}} \frac{2 u-1}{(u+b) u(u-1)} \mathrm{d} u .
$$

The latter integral is easily computed and the result gives

$$
\widehat{T}_{\text {peak }}=-\frac{1}{m}\left[-\frac{2 b+1}{b(b+1)} \ln (u+b)+\frac{1}{b} \ln (u)+\frac{1}{b+1} \ln (u-1)\right]_{h_{3}\left(v_{-}\right)}^{u_{\text {sldn }}} .
$$

The asymptotic AP duration is now slightly modified as

$$
\begin{aligned}
\widehat{T}_{\mathrm{AP}} & =\widehat{T}_{\text {peak }}+\tau_{\text {sclose }} \ln \left(\frac{\tau_{\text {out }} h_{3}^{-1}\left(u_{\text {sldn }}\right)}{4 \tau_{\text {in }}}\right) \\
& =\widehat{T}_{\text {peak }}-\tau_{\text {sclose }} \ln \left(4 u_{\text {sldn }}\left(1-u_{\text {sldn }}\right)\right)
\end{aligned}
$$

The asymptotic recovery duration associated to phase IV can be derived with assumptions similar to those of phase II. The dimensional scaling is based on the recovery period, then $T=T_{g}$ and equation (4.4) reduces again to $f(u, v)=0$ to leading order. The system for the recovery variable reads as

$$
\frac{\partial v}{\partial t}=\frac{T}{T_{g}} g\left(h_{1}(v), v\right), v(0)=v_{*},
$$

with $u=h_{1}(v)$ for the asymptotic solution to be continuous (matching condition with phase III).

For the original MS model, $v$ increases and the solution goes back to the stable point $(0,1)$ so the time to reach this equilibrium is infinitely long. If a region of the domain is paced at a certain value of $v$, say $v_{\text {pace }}$, the recovery time for the MS model can be approximated by

$$
\widehat{T}_{\text {rec }}=\tau_{\text {open }} \ln \left(\frac{\frac{4 \tau_{\text {in }}}{\tau_{\text {out }}}-1}{v_{\text {pace }}-1}\right) .
$$

The last estimate is calculated as for $\widehat{T}_{\mathrm{AP}}$ in phase II. 
Our definition of the asymptotic recovery time $\widehat{T}_{\text {rec }}$ is the interval between times where the transmembrane potential reaches the resting state $(u=0)$ and the recovery variable $v$ equals $v_{\text {pace. }}$ In this case, the asymptotic recovery time $\widehat{T}_{\text {rec }}$ corresponds to the diastolic interval between two AP.

We could have chosen $v=v_{t h, r e c}$, where $v_{t h, r e c}$ is the recovery (in fact refractory) threshold at which excitation is again possible with strong enough stimulation. Phase IV then corresponds to the end of the refractory period past return to equilibrium potential. Even for simple two-variable models, it may be hard to properly define or assess this recovery threshold. For the MS model, the recovery threshold is dependent on $v$. Equation (4.19) then allows to know which $\tau_{\text {open }}$ to choose for having a particular recovery interval. For other more complex models, it may not be possible to determine the recovery threshold as easily as for the MS model, e.g. where the recovery threshold could depend on the state of many variables interacting together. Moreover the threshold is dependent on the strength of the stimulation current.

When $a \neq 0$, the upper bound for $v$ in this phase cannot be found exactly unless an analysis of the periodic solution is performed. This is not going to be done just because doing such a laborious analysis is pointless considering the objective: evaluating the following

$$
\widehat{T}_{\text {rec, a }}=\int_{v_{*}}^{v_{\text {up }, \text { periodic }}} \frac{1}{g\left(h_{1}(v), v\right)} \mathrm{d} v,
$$

where $v_{\text {up,periodic }}$ is the value of $v$ during the upstroke of an asymptotic periodic solution. One can however

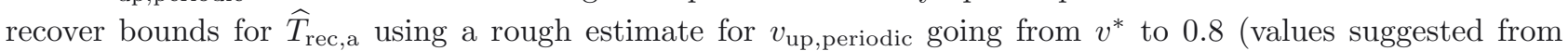
numerical simulations). The dimensional time for this phase is then

$$
\int_{v_{*}}^{v^{*}} \frac{1-v}{\tau_{\text {open }}} \mathrm{d} v \lesssim T_{\text {rec }, \mathrm{a}} \lesssim \int_{v_{*}}^{0.8} \frac{1-v}{\tau_{\text {open }}} \mathrm{d} v
$$

as for the modified MS model, $u<u_{\text {gate }}$ and then $g\left(h_{1}(v), v\right)=(1-v) / \tau_{\text {open. }}$. With the dimensional values of $\tau$ given in Section 2.2 , the recovery time can be estimated with $122 \lesssim T_{\text {rec,a }} \lesssim 211 \mathrm{~ms}$.

\subsubsection{The asymptotic space scales and speed}

Isolating $\widehat{L}_{\text {up }}$ in equation (4.13), an expression for the asymptotic upstroke width is obtained:

$$
\widehat{L}_{\text {up }}=k_{\text {up }} \sqrt{\widehat{T}_{\text {up }}} \sqrt{\frac{\sigma}{C_{\mathrm{m} \chi}}} .
$$

In the right hand side, the factor $k_{\text {up }} \sqrt{\widehat{T}_{\text {up }}}$ does not depend on the diffusion constant $\sigma /\left(C_{\mathrm{m}} \chi\right)$. It depends only on the parameters of the ionic model. Substituting $\widehat{T}_{\text {up }}$ from (4.15), expressions for the asymptotic length and speed of the wave are easily derived, such as

$$
\widehat{c}=\frac{\widehat{L}_{\mathrm{up}}}{\widehat{T}_{\mathrm{up}}}=-\frac{F_{\mathrm{AB}} \widehat{L}_{\mathrm{up}}}{h_{3}^{2}\left(v_{-}\right)}=k_{\mathrm{up}} \frac{\sqrt{-F_{\mathrm{AB}}\left(v_{-}\right)}}{h_{3}\left(v_{-}\right)} \sqrt{\frac{\sigma}{C_{\mathrm{m}} \chi}} .
$$

Similarly, the other asymptotic widths are given by

$$
\widehat{L}_{i}=k_{i} \sqrt{\frac{\widehat{T}_{i} \sigma}{C_{\mathrm{m}} \chi}} \quad \text { for } \quad i=\text { down, AP, rec. }
$$

\subsection{Using the asymptotic solution to predict the numerical solution}

The aim of this section is to see how the asymptotic times $\widehat{T}$ and lengths $\widehat{L}$ can be used to predict the dimensional times and lengths for the various phases of the numerical solution of the bidomain model with MS ion kinetics. 
We first link the upstroke time $T_{\text {up }}$ required for the rise of the numerical solution $u$ from $u_{\text {gate }}$ to $h_{3}\left(v_{-}\right)$to the asymptotic upstroke time $\widehat{T}_{\text {up }}$ as given by equation (4.15). This asymptotic time assumes that the upstroke occurs linearly at a constant $v=v_{-}$, which makes the values of $\widehat{T}_{\text {up }}$ a bit off the value of $T_{\text {up }}$ though the behaviour with respect to the parameters remains satisfactory. For $\widehat{T}_{\text {up }}$ to be convenient to estimate $T_{\text {up }}$, it can be scaled with a constant $k_{\mathrm{AB}}>0$ as

$$
T_{\mathrm{up}} \simeq k_{\mathrm{AB}} \widehat{T}_{\mathrm{up}}=k_{\mathrm{AB}} \frac{h_{3}^{2}\left(v_{-}\right)}{\left|F_{\mathrm{AB}}\left(v_{-}\right)\right|},
$$

where the constant $k_{\mathrm{AB}}$ can be found using a numerical simulation for a given set of $\left\{\tau_{\text {in }}, \tau_{\text {out }}\right\}$.

In Section 4.4.1 we obtained two other expressions for $\widehat{T}_{\text {up }}$, one which was based on an exponential upstroke profile for the travelling wave. At the end, changing the approximate upstroke profile, hence the expression for $\widehat{T}_{\text {up }}$, can be compensated by adjusting the scaling constant $k_{\mathrm{AB}}$. For the sake of simplicity, it turns out that the linear rise is a good choice: simple calculations and satisfactory predictions. See Section 5 for examples showing that this approximation is good enough to be used for the prediction of $T_{\text {up }}$ in the heart.

For simulations with spatial propagation, the constant $k_{\mathrm{AB}}$ can be calculated from a single simulation in 1D, using a set of parameters denoted by $\left\{\tau_{\text {in }}, \tau_{\text {out }}\right\}_{0}$. If $\left\{\tau_{\text {in }}, \tau_{\text {out }}\right\}$ changes for any reason (for example, from one tissue of the heart to an other, where the AP is different) but in a way that the new upstroke profile is simply a rescaling of the profile set by the value $\left\{\tau_{\text {in }}, \tau_{\text {out }}\right\}_{0}$, then the constant $k_{\mathrm{AB}}$ remains unchanged. Basically, if the new profile is a scaling of the profile for $\left\{\tau_{\text {in }}, \tau_{\text {out }}\right\}_{0}$, then the linear profile used to estimate the new upstroke profile is scaled in the same way, and the quality of fit between the exact and approximate linear upstroke profiles is unchanged. This scaling argument is nearly valid for instance when $\left\{\tau_{\text {in }}, \tau_{\text {out }}\right\}$ remains in a certain neighbourhood of $\left\{\tau_{\text {in }}, \tau_{\text {out }}\right\}_{0}$.

We will see later that this way of estimating $T_{\text {up }}$ works in a reliable way, as long as $u_{\text {gate }}$ remains the same. Changing the value of $u_{\text {gate }}$ is equivalent to changing the threshold of the upstroke. Consequently, it could modify the upstroke profile, then the integral of its derivative and so the scaling factor $k_{\mathrm{AB}}$. Section 5 presents cases where $k_{\mathrm{AB}}$ has to be recalculated.

The dimensional downstroke duration can also be predicted with

$$
T_{\text {down }} \simeq k_{C D} \widehat{T}_{\text {down }}=12 k_{C D} \tau_{\text {out }},
$$

where the constant $k_{C D}>0$ arises from the same arguments as for $k_{\mathrm{AB}}$. Assuming that the phase III occurs for a constant $v=v_{*}$ is not as nearly true as for the upstroke. However, the scaling constant $k_{C D}$ is enough to compensate for the approximations used to find $\widehat{T}_{\text {down }}$.

Equations (4.21) and (4.22) build a relation $\xi$ between two model parameters and two asymptotic solution features for phases I and III:

$$
\xi:\left(\tau_{\text {in }}, \tau_{\text {out }}\right) \rightarrow\left(k_{\mathrm{AB}} \widehat{T}_{\text {up }}, k_{C D} \widehat{T}_{\text {down }}\right) .
$$

For solution features $T_{\mathrm{up}}$ and $T_{\text {down }}$ of physiological relevance, it turns out that $\xi$ is bijective. Figures 8 and 9 illustrate the mapping $\xi$ and how well it represents the dimensional times $T_{\mathrm{up}}$ and $T_{\mathrm{down}}$ for numerical solutions.

For phase II, the action potential duration $T_{\mathrm{AP}}$ is well approximated by

$$
T_{\mathrm{AP}} \approx \widehat{T}_{\mathrm{AP}}
$$

without the need for any scaling constant. Both the asymptotic and numerical solutions follow very closely the nullcline $f(u, v)=0$ and no assumption is made on the shape of the wave, hence the quality of fit of the asymptotic solution to the exact solution. Similarly, $T_{\text {peak }} \approx \widehat{T}_{\text {peak. }}$. Figures 10 and 11 illustrate the bijection between $\left(\tau_{\text {sclose }}, \tau_{\text {fclose }}\right)$ and $\left(\widehat{T}_{\mathrm{AP}}, \widehat{T}_{\text {peak }}\right)$.

For phase IV, the recovery time can be predicted as long as the next wave is paced at a given value $v_{\text {pace }}$ and $T_{\text {rec }} \approx \widehat{T}_{\text {rec }}$ with $\widehat{T}_{\text {rec }}$ given by equation (4.19). For waves paced at various values of $v_{\text {pace }}$, the asymptotic analysis provides very good estimates (see Sect. 4.6). 


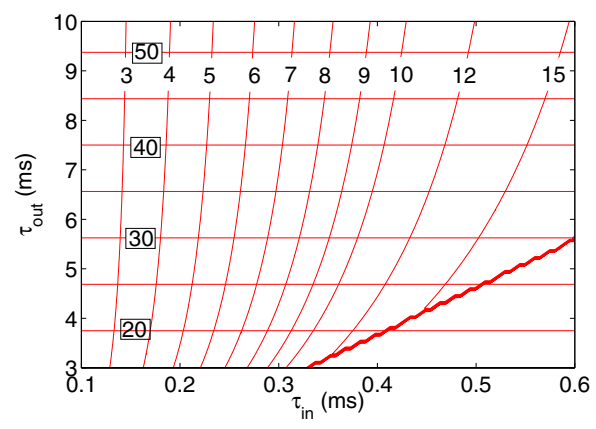

FIGURE 8. Asymptotic approximations of the durations $\left(k_{\mathrm{AB}} \widehat{T}_{\mathrm{up}}, k_{C D} \widehat{T}_{\text {down }}\right)$ versus $\left(\tau_{\mathrm{in}}, \tau_{\text {out }}\right)$ for $v_{-}=1, k_{\mathrm{AB}}=2.15$ and $k_{C D}=0.45$. Level sets for $\widehat{T}_{\text {up }}$ are nearly vertical (plain labels) and level sets for $\widehat{T}_{\text {down }}$ are horizontal (boxed labels).

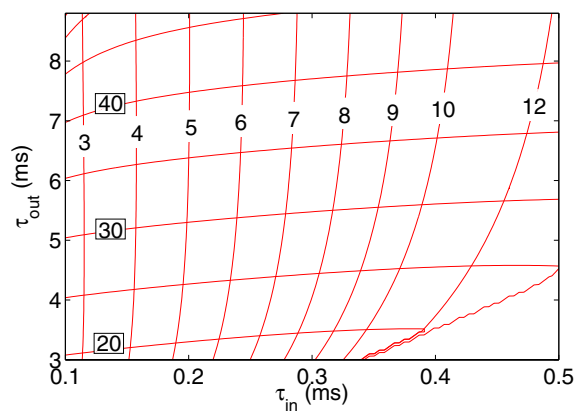

Figure 9. 1D numerical solution. ( $\left.T_{\mathrm{up}}, T_{\text {down }}\right)$ versus $\left(\tau_{\mathrm{in}}, \tau_{\text {out }}\right)$ for $v_{-}=1, \tau_{\text {open }}=130 \mathrm{~ms}$, $\tau_{\text {close }}=150 \mathrm{~ms}$. Level sets for $\widehat{T}_{\text {up }}$ are nearly vertical (plain labels) and level sets for $\widehat{T}_{\text {down }}$ are nearly horizontal (boxed labels). Note that the ranges for the $\tau_{\text {in }}$ and $\tau_{\text {out }}$ axis are not the same as in Figure 8.

The numerical upstroke length is predicted using

$$
L_{\mathrm{up}} \simeq \sqrt{k_{\mathrm{AB}}} \widehat{L}_{\mathrm{up}}=k_{\mathrm{up}} \sqrt{\frac{T_{\mathrm{up}} \sigma}{C_{\mathrm{m}} \chi}} .
$$

Whenever the parameters of the MS model and $v_{-}$are kept fixed, $k_{\text {up }}$ remains constant. As stated in Section 4.3.2, the dimensionless number associated with phase I, namely $N_{\text {up }}=\sigma T_{\text {up }} /\left(C_{\mathrm{m}} \chi L_{\text {up }}^{2}\right) \simeq 1 / k_{\text {up }}^{2}$, also remains constant. The numerical results predicted by equation (4.24) are illustrated in Figure 6a. To fit the asymptotic behaviour to the data, the dimensionless number was $N_{\text {up }}=0.1149$.

The speed of the numerical solution can be predicted from the asymptotic speed using

$$
c=\frac{L_{\mathrm{up}}}{T_{\mathrm{up}}} \simeq \frac{\sqrt{k_{\mathrm{AB}}} \widehat{L}_{\mathrm{up}}}{k_{\mathrm{AB}} \widehat{T}_{\mathrm{up}}}=\frac{1}{\sqrt{k_{\mathrm{AB}}}} \widehat{c}
$$

The numerical results predicted are illustrated in Figure 6b, still with $N_{\text {up }}=0.1149$.

Remark 4.8 (Precisions about the numerical simulations). The numerical values $T_{\text {up }}$ and $L_{\text {up }}$ are averaged over the total time of the simulation. The time and space discretization was fine enough so that the solution 


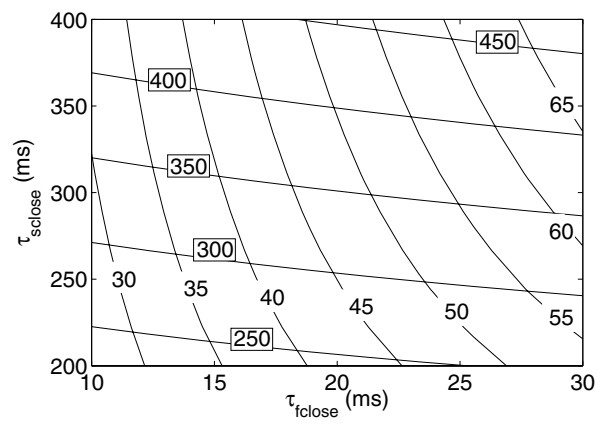

FIGURE 10. Asymptotic approximations of the durations $\left(\widehat{T}_{\mathrm{AP}}, \widehat{T}_{\text {peak }}\right)$ versus $\left(\tau_{\text {fclose }}, \tau_{\text {sclose }}\right)$ for $v_{-}=1, \tau_{\text {in }}=0.3 \mathrm{~ms}, \tau_{\text {out }}=6 \mathrm{~ms}$. Level sets for $\widehat{T}_{\text {peak }}$ are nearly vertical (plain labels) and level sets for $\widehat{T}_{\mathrm{AP}}$ are nearly horizontal (boxed labels).

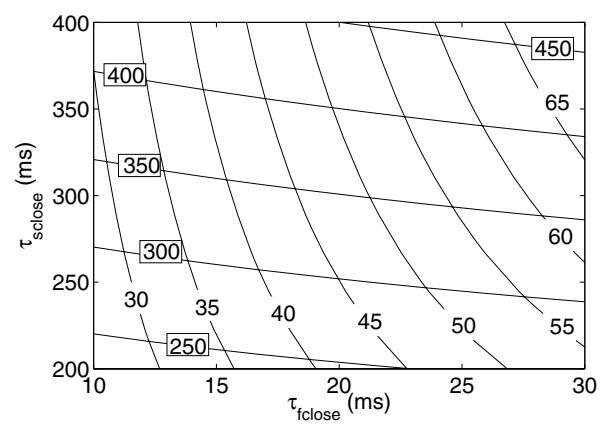

FigURE 11. 1D numerical solution. $\left(T_{\mathrm{AP}}, T_{\text {peak }}\right)$ versus $\left(\tau_{\text {fclose }}, \tau_{\text {sclose }}\right)$ for $v_{-}=1, \tau_{\text {in }}=0.3 \mathrm{~ms}$, $\tau_{\text {out }}=6 \mathrm{~ms}, \tau_{\text {open }}=130 \mathrm{~ms}, \tau_{\text {close }}=150 \mathrm{~ms}$. Level sets for $\widehat{T}_{\text {peak }}$ are nearly vertical (plain labels) and level sets for $\widehat{T}_{\mathrm{AP}}$ are nearly horizontal (boxed labels).

was converged in time and space with less than $1 \%$ of variation on $T_{\mathrm{up}}, L_{\mathrm{up}}, T_{\mathrm{AP}}, L_{\mathrm{AP}}$ and speed compared to an overly precise solution.

Similarly, the value of the dimensionless number $N_{\text {down }}=\left(T_{\text {down }} \sigma\right) /\left(C_{\mathrm{m}} \chi L_{\text {down }}^{2}\right)$ associated to phase III is nearly constant and can be found using the same 1D simulation. The downstroke width can estimated as well using equation (4.22) and

$$
L_{\text {down }} \simeq \sqrt{k_{C D}} \widehat{L}_{\text {down }}=\frac{1}{\sqrt{N_{\text {down }}}} \sqrt{\frac{k_{C D} \widehat{T}_{\text {down }} \sigma}{C_{\mathrm{m}} \chi}} .
$$

The validity of the approximation is striking. Using the same $1 \mathrm{D}$ simulation, one gets $k_{C D}=0.45$ and $N_{\text {down }}=$ 0.02601. $T_{\text {down }}$ and $L_{\text {down }}$ could be plotted versus $\sigma /\left(C_{\mathrm{m}} \chi\right)$. The figure is very similar to Figure 6 and will not be showed to avoid redundancy.

The behaviour of the AP length is asymptotically of the form

$$
L_{\mathrm{AP}} \simeq \frac{1}{\sqrt{N_{\mathrm{AP}}}} \sqrt{\frac{\widehat{T}_{\mathrm{AP}} \sigma}{C_{\mathrm{m} \chi}}} .
$$




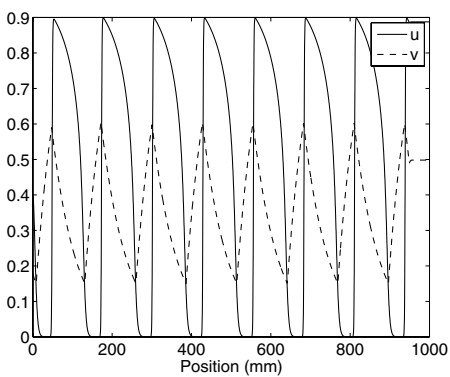

(a) Wave train paced at $v_{-}=0.6$.

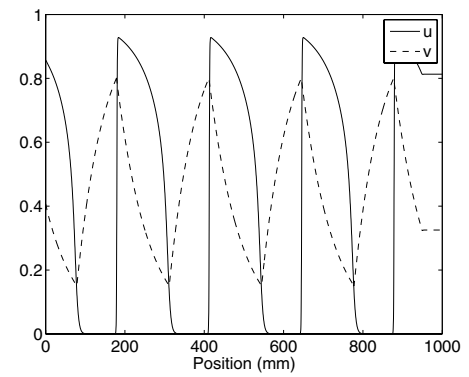

(b) Wave train paced at $v_{-}=0.8$.

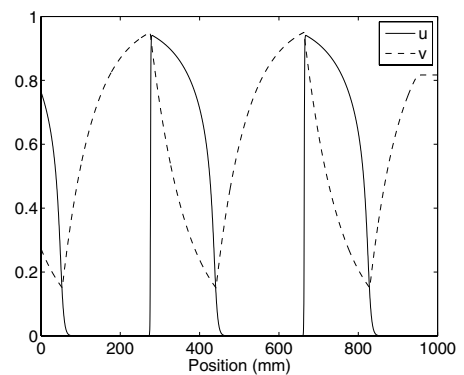

(c) Wave train paced at $v_{-}=0.95$.

Figure 12. The simulations are calculated over $5000 \mathrm{~ms}$. The dimensionless number $N$ is that of Adim3.

The dimensionless number associated to the phase II is $N_{\mathrm{AP}}=3.3029 \times 10^{-3}$ and is found using the same 1D numerical simulation as the one used to fit the upstroke length. Again, the behaviour of the action potential duration and width can be predicted accurately.

Finally, the recovery length can be found by fixing the dimensionless number for this phase (using again the same simulation as the one used to fit the upstroke width). Again, the asymptotic results fit almost perfectly the numerical solutions.

\subsection{Periodic excitations}

In the following, relations obtained from the asymptotic analysis are going to be used to predict what happens to the solution of the MS model under periodic pacing. When action potentials are paced at a high frequency, the recovery variable $v$ cannot reach the equilibrium value $v=1$ between each pacing, hence $v_{-}<1$ in the asymptotic solution. Several features of the asymptotic solution, such as the wave speed (Eq. (4.20)) and the upstroke duration (Eq. (4.15)), depend on the state $v_{-}$. Consequently, the action potential differs for an isolated wave compared to closely paced waves. This kind of phenomenon occurs for instance in heart beats for exercising subjects and in pathological situations like arrhythmias and fibrillation. The last example is often modelled with spiral waves (e.g. see [19]), where wave fronts propagate close to each other.

In the numerical simulations used to illustrate periodic wave train, a new wave is initiated in a pacing region every time the recovery variable $v$ reaches a prescribed value $v_{\text {pace }}=v_{-}$in that region. A sequence of waves is generated, and following a transient period, the wave train is stable. All the waves then have the same speed, action potential duration, recovery time, etc. The dependencies on $v_{-}$are analysed once a stable wave train is obtained.

Figure 12 illustrates stable periodic wave train for three values of $v_{-}$. One can see that the distance between waves and the wave length decrease with an increasing pacing frequency. Figure 13 shows that the asymptotic analysis reliably predicts $T_{\mathrm{AP}}$ and $T_{\text {rec }}$ for wave trains at different $v_{-}$. Predicting $T_{\text {up }}$ for wave trains is more difficult because of the difficulty in adjusting the scaling factor $k_{\mathrm{AB}}$ for varying $v_{-}$. The approximation $\widehat{T}_{\mathrm{up}}$ may be up to $35 \%$ off $T_{\text {up }}$ near $v_{-}=0.6$. The approximate speed $\widehat{c}$ is relatively reliable since in the worst case near $v_{-}=0.6, \widehat{c}$ is less than $6 \%$ off $c$. The approximation for $T_{\text {down }}$ looks poor because $\widehat{T}_{\text {down }}$ does not catch the trend for $v_{-}$, though it should be noted that $T_{\text {down }}$ varies for less than $4 \%$ from $v_{-}=0.6$ to $v_{-}=1$. Globally, the asymptotic approximation describing the behaviour of wave trains is very good as long as the prediction of the upstroke duration is. 


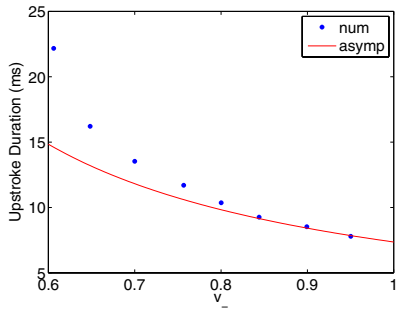

(a) Upstroke duration

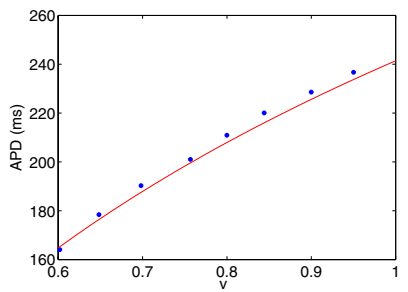

(b) Action potential duration

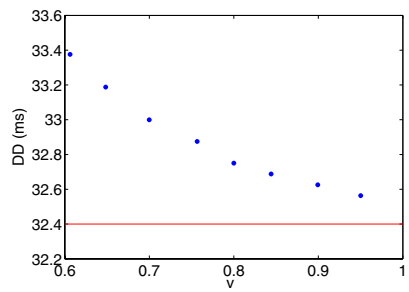

(c) Downstroke duration

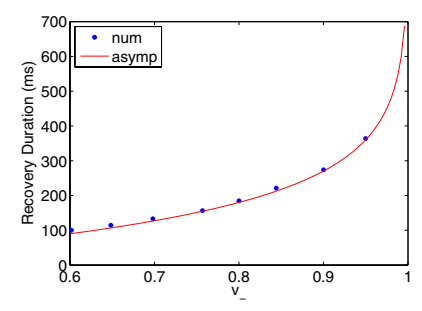

(d) Recovery duration

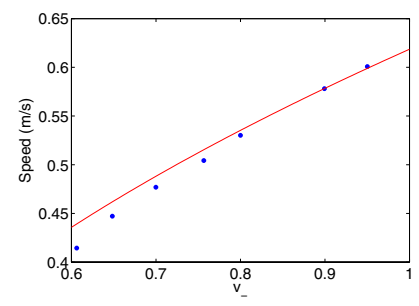

(e) Speed of the wave train

FiguRe 13. Asymptotic estimates (continuous line) and numerical values (dots) of wave train AP features as a function of the pacing threshold $v_{-}$.

\subsection{Predicting the restitution curves}

Using equation (4.19), the restitution curves can be plotted for the upstroke duration, the action potential, the downstroke duration and the speed. Figure 14 shows the upstroke duration $T_{u p, n+1}$ of a wave following a recovery duration of $T_{\mathrm{rec}, \mathrm{n}}$ (here refers to the diastolic interval), same idea for the other features.

\section{Modelling Physiological AP propagating in VARious tissues of the HeART}

This section is devoted to the application of the asymptotic analysis to model various AP with the aid of the one-to-one relation

$$
\left\{\tau_{\text {in }}, \tau_{\text {out }}, \tau_{\text {open }}, \tau_{\text {close }}, N\right\} \rightarrow\left\{\widehat{T}_{\text {up }}, \widehat{T}_{\text {AP }}, \widehat{T}_{\text {down }}, \widehat{T}_{\text {rec }}, c\right\} .
$$

In the human heart, there are different tissues with different AP propagating at different speeds. It is important to notice that the exact shapes of the AP in various tissues cannot be perfectly replicated, but the durations of every phase are in agreement. A Purkinje fibre AP as well as a ventricle AP of a healthy human heart are going to be modelled in order to prove the efficiency of our asymptotic analysis.

The very first step is to simulate one AP in $1 \mathrm{D}$ using a given set of parameters $\left\{u_{\text {gate }}, \tau_{\text {in }}, \tau_{\text {out }}, \tau_{\text {open }}, \tau_{\text {close }}\right\}$. Note that the same $u_{\text {gate }}$ has to be used for every simulation unless a new $1 \mathrm{D}$ simulation is computed every time $u_{\text {gate }}$ is changed. Using the results of the $1 \mathrm{D}$ simulation, the constants $k_{\mathrm{AB}}$ and $k_{C D}$ can be fixed to adjust the asymptotic predictions with the numerical solution at such $u_{\text {gate }}$.

The second step is to set the parameters of the MS model to recover the desired time scales of the (Purkinje or ventricle) AP. From equations (4.15)-(4.19), we have the following relations:

$$
\begin{aligned}
& T_{\text {up }} \simeq k_{\mathrm{AB}} \widehat{T}_{\text {up }}\left(\tau_{\text {in }}, \tau_{\text {out }}\right) ; \\
& T_{\mathrm{AP}} \simeq \widehat{T}_{\mathrm{AP}}\left(\tau_{\text {close }}, \tau_{\text {in }}, \tau_{\text {out }}\right) ; \\
& T_{\text {down }} \simeq k_{C D} \widehat{T}_{\text {down }}\left(\tau_{\text {out }}\right) ; \\
& T_{\text {rec }} \simeq \widehat{T}_{\text {rec }}\left(\tau_{\text {open }}, \tau_{\text {in }}, \tau_{\text {out }}\right) .
\end{aligned}
$$




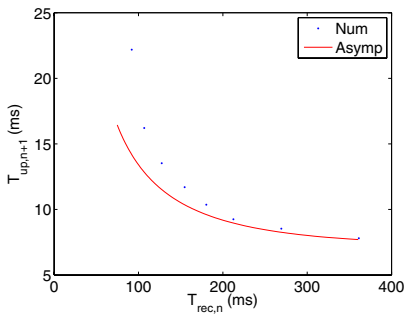

(a) Upstroke duration $T_{u p, n+1}$ vs. $T_{\text {rec,n }}$.

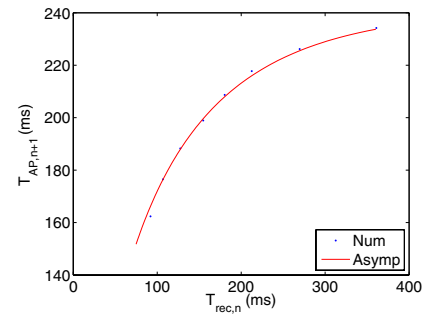

(b) Action potential duration

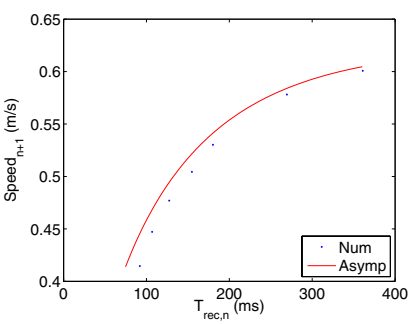

(d) Speed of the wave

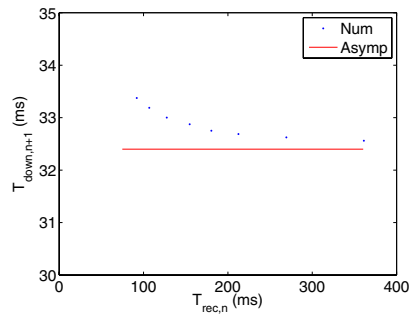

(c) Downstroke duration

Figure 14. Restitution curves obtained through asymptotic estimates (continuous line) and numerical computations (dots).

The order in which the parameters of the MS model have to be fixed is obvious: $\tau_{\text {out }}$ from $\widehat{T}_{\text {down }}$, followed by $\tau_{\text {in }}$ from $\widehat{T}_{\text {up }}$, followed by $\tau_{\text {open }}$ and $\tau_{\text {close }}$ from $\widehat{T}_{\text {rec }}$ and $\widehat{T}_{\text {AP }}$, respectively. If for any reason the overshoot is important in the simulation, one more relation is considered

$$
T_{\text {peak }} \simeq \widehat{T}_{\text {peak }}\left(u_{\text {sldn }}, \tau_{\text {in }}, \tau_{\text {out }}, \tau_{\text {fclose }}, \tau_{\text {sclose }}\right) .
$$

The parameters are fixed in the following order: $u_{\text {sldn }}$ from the overshoot height, followed by the same steps as without the overshoot and finally, $\tau_{\text {fclose }}$ from the overshoot duration.

The third step is to impose the speed of propagation by fixing the diffusion constant $\sigma /\left(C_{\mathrm{m}} \chi\right)$ in the bidomain model from equations (4.20) and (4.25). Using the results of the 1D simulation, the dimensionless numbers $N_{\text {up }}$, $N_{\text {down }}, N_{\mathrm{AP}}$ and $N_{\text {rec }}$ are recovered from the time and length scales of the various phases of the numerical solution. The space scales $L_{\mathrm{up}}, L_{\mathrm{down}}, L_{\mathrm{AP}}$ and $L_{\mathrm{rec}}$ are then deduced.

Finally, the characteristic potential $V_{\mathrm{m}}$ is fixed so that the AP sweeps an appropriate range in Volts. Any characteristic time $T$ and length $L$ can be chosen as long as the domain is properly scaled. For instance, if a numerical simulation is done on a mesh of a human heart, $L$ has to be fixed so that the heart has the right size and $T$ can be chosen arbitrarily. The nondimensional values of $\tau$ 's in the MS model will have to be adjusted consequently because the original MS model is designed for $T=1 \mathrm{~ms}$.

\subsection{Ventricle}

In the intent of reproducing the ventricle AP, the first numerical simulation uses parameters such that the AP is physiological, i.e. has time scales and speed of real patient data.

In order to see the entire cycle with the four phases in the numerical simulation, the interval $\left[0, t_{\max }\right]$ over which the simulation is done is chosen such that $t_{\max }>\widehat{T}_{\mathrm{up}}+\widehat{T}_{\mathrm{AP}}+\widehat{T}_{\text {down }}+\widehat{T}_{\text {rec }}$. The size of the domain has to be larger than $\widehat{c} t_{\max }$. 
TABLE 3. Model parameters to get the asymptotic - -quantities for a physiological ventricle.

\begin{tabular}{|c|c|c|c|c|c|c|}
\hline Phase & Duration & $\begin{array}{c}\widehat{T} \\
(\mathrm{~ms})\end{array}$ & \multicolumn{2}{|c|}{$\begin{array}{l}\text { MS parameters } \\
(\mathrm{ms})\end{array}$} & $\begin{array}{c}\mathrm{T} \\
(\mathrm{ms})\end{array}$ & $\begin{array}{c}|\widehat{T}-T| / \widehat{T} \\
(\%)\end{array}$ \\
\hline I & Upstroke & 8 & $\tau_{\text {in }}$ & 0.3150 & 7.9750 & 0.313 \\
\hline II & $\mathrm{AP}$ & 250 & $\tau_{\text {close }}$ & 168.50 & 260.93 & 4.38 \\
\hline III & Downstroke & 30 & $\tau_{\text {out }}$ & 5.5556 & 31.196 & 4.00 \\
\hline IV & Recovery & 260 & $\tau_{\text {open }}$ & 94.942 & 257.41 & 0.996 \\
\hline Phase & Speed & $\begin{array}{c}\widehat{c} \\
(\mathrm{~m} / \mathrm{s})\end{array}$ & \multicolumn{2}{|c|}{$\begin{array}{l}\text { Bidomain parameter } \\
\text { (dimensionless) }\end{array}$} & $\begin{array}{c}c \\
(\mathrm{~m} / \mathrm{s})\end{array}$ & $\begin{array}{c}|\widehat{c}-c| / \widehat{c} \\
(\%)\end{array}$ \\
\hline I & & 0.5 & $\mathrm{~N}$ & $1.8508 \mathrm{e}-04$ & 0.4368 & 12.6 \\
\hline
\end{tabular}

TABLE 4. Model parameters to get the asymptotic - -quantities for a physiological Purkinje system.

\begin{tabular}{|c|c|c|c|c|c|c|}
\hline Phase & Duration & $\begin{array}{c}\widehat{T} \\
(\mathrm{~ms})\end{array}$ & \multicolumn{2}{|c|}{$\begin{array}{c}\text { MS parameters } \\
(\mathrm{ms})\end{array}$} & $\begin{array}{c}\mathrm{T} \\
(\mathrm{ms})\end{array}$ & $\begin{array}{c}|\widehat{T}-T| / \widehat{T} \\
(\%)\end{array}$ \\
\hline I & Upstroke & 8 & $\tau_{\text {in }}$ & 0.3590 & 8.77 & 9.7 \\
\hline II & AP & 380 & $\tau_{\text {close }}$ & 178.73 & 401.12 & 5.6 \\
\hline III & Downstroke & 65 & $\tau_{\text {out }}$ & 12.037 & 61.54 & 5.4 \\
\hline IV & Recovery & 320 & $\tau_{\text {open }}$ & 111.55 & 305.29 & 4.6 \\
\hline Phase & Speed & $\begin{array}{c}\widehat{\widehat{c}} \\
(\mathrm{~m} / \mathrm{s})\end{array}$ & \multicolumn{2}{|c|}{$\begin{array}{l}\text { Bidomain parameter } \\
\text { (dimensionless) }\end{array}$} & $\begin{array}{c}c \\
(\mathrm{~m} / \mathrm{s})\end{array}$ & $\begin{array}{c}|\widehat{c}-c| / \widehat{c} \\
(\%)\end{array}$ \\
\hline I & & 1.8 & $\mathrm{~N}$ & 0.0024 & 1.65 & 8.4 \\
\hline
\end{tabular}

The numerical solution is performed on a domain of $1000 \mathrm{~mm}$ over $1000 \mathrm{~ms}$. A forward Euler time-stepping scheme is used with 80000 time steps and a finite difference method is used in space with 4000 dofs. A characteristic time $T=1 \mathrm{~ms}$ and length $L=1 \mathrm{~mm}$ are used for the nondimensionalisation. $u_{\text {gate }}=0.13$ and the scaling constants $k_{\mathrm{AB}}, k_{C D}$ as well as the nondimensional numbers of every phase are the same as those used in Section 4.5. The results of the asymptotic predictions are presented in Table 3 and the numerical simulation gives a solution with no more than $5 \%$ off the predicted time scales. Note that $c$ is $12.6 \%$ off its asymptotic prediction. As $c$ varies like $\sqrt{N}$, the number $N$ is readily adjustable so that the desired speed can be obtained.

\subsection{Purkinje fibres}

This is an example where the AP has time scales of the order of magnitude of those of the ventricle AP. However, the speed of propagation is much larger. The scaling constants $k_{\mathrm{AB}}, k_{C D}$ as well as the dimensionless numbers of every phase are the same as those used for the simulation of the ventricle AP. The numerical solution is computed on a domain of $2000 \mathrm{~mm}$-long over $1000 \mathrm{~ms}$, using a forward Euler time-stepping scheme with 100,000 time steps and 8000 dofs. The results of the asymptotic predictions are presented in Table 4 and the numerical simulation gives a solution with no more than $10 \%$ off the predicted time scales and speed.

\subsection{Precautions for a narrower upstroke}

For a very steep upstroke lasting only $1 \mathrm{~ms}, \tau_{\text {in }}$ has to be reduced to 0.0827 . This affects considerably the phase space. For a complete cycle to occur during the simulation, $u_{\text {gate }}$ has to be reduced otherwise the domain gets re-depolarised prematurely. We used $u_{\text {gate }}=0.005$. The numerical solution is performed on a domain of $1000 \mathrm{~mm}$-long over $1000 \mathrm{~ms}$, with 100000 time steps and 4000 dofs. The scaling constants $k_{\mathrm{AB}}, k_{C D}$ had to be re-calculated. The $\tau$ 's are obtained using the results of a numerical simulation with the $\widehat{T}$ calculated with 
TABLE 5. Model parameters to get the asymptotic - -quantities for a very steep upstroke in a physiological Purkinje system.

\begin{tabular}{|l|c|c|c|c|c|c|}
\hline Phase & Duration & $\begin{array}{c}\widehat{T} \\
(\mathrm{~ms})\end{array}$ & \multicolumn{2}{|c|}{$\begin{array}{c}\text { MS parameters } \\
(\mathrm{ms})\end{array}$} & $\begin{array}{c}\mathrm{T} \\
(\mathrm{ms})\end{array}$ & $\begin{array}{c}|\widehat{T}-T| / \widehat{T} \\
(\%)\end{array}$ \\
\hline I & Upstroke & 1 & $\tau_{\text {in }}$ & 0.02 & 0.9554 & 4.46 \\
II & AP & 380 & $\tau_{\text {close }}$ & 71.026 & 407.68 & 7.28 \\
III & Downstroke & 65 & $\tau_{\text {out }}$ & 16.852 & 58.940 & 9.32 \\
IV & Recovery & 320 & $\tau_{\text {open }}$ & 106.989 & 360.05 & 12.52 \\
\hline Phase & Speed & $\widehat{c}$ & \multicolumn{2}{c|}{ Bidomain parameter } & $c$ & $|\widehat{c}-c| / \widehat{c}$ \\
& & $(\mathrm{~m} / \mathrm{s})$ & \multicolumn{2}{c|}{ (dimensionless) } & $(\mathrm{m} / \mathrm{s})$ & $(\%)$ \\
\hline I & & 1.8 & $\mathrm{~N}$ & $1.6175 \mathrm{e}-04$ & 1.7975 & 0.03 \\
\hline
\end{tabular}

the constants $k_{\mathrm{AB}}$ and $k_{C D}$ of $u_{\text {gate }}=0.13$. Obviously this preliminary $1 \mathrm{D}$ simulation gives wrong results in time scales and speed, but it is essential to find $k_{\mathrm{AB}}, k_{C D}$ as well as the dimensionless numbers of every phase associated with $u_{\text {gate }}=0.005$. Once these constants are found, a new numerical simulation is performed. The results of the asymptotic predictions for a Purkinje fibre are presented in Table 5. The numerical simulation gives a solution with no more than $13 \%$ off the predicted long time scales and no more than $10 \%$ off the predicted short time scales. The speed is very well predicted.

Remark 5.1. Even if this study is based on one-dimensional results, it can be also applied in 3D where the anisotropic conductivity properties of the heart tissue are known. For a planar or nearly planar wavefront propagating in the direction $n$ at speed $c$, we have $u(x, t)=u(x \cdot n, t)$ and we are brought to a local onedimensional problem. The conductivities in the direction $n$ are $s_{i, e}(n)=n^{T} \sigma_{i, e} n$ and the elliptic operators simplify to $\nabla \cdot \sigma_{i, e} \nabla u(x, t)=s_{i, e} \partial_{\eta \eta} u(x \cdot n, t)$, where $\partial_{\eta \eta}$ denotes the second order partial derivative along $\eta=x \cdot n$. The results of our asymptotic analysis can thus be applied. For curved wavefronts or conductivity tensors with substantial spatial inhomogeneities, extension of our work is required for instance borrowing ideas from eikonal-curvature or eikonal-diffusion equations $[8,18,31]$.

\section{Conclusion}

The dimensional and asymptotic analysis presented here provides the steps to control the solution in a local region of constant conductivity. It makes it possible to match the time/space scales and speed of any AP that is to say the shape of the AP and its propagation. This can be done because explicit relations between the physiological quantities and the model parameters are derived. The application of the method was done successfully on two very different tissues of the heart, the ventricle and the Purkinje fibres. Even when time scales differ by many orders of magnitude in the same AP, the reliability of the method with the MS phenomenological model has been demonstrated.

This method facilitates the integrative modelling of a complete human heart with tissue-specific ionic models. It indicates that using a single phenomenological model for the membrane potential is enough to compute propagation at the tissue scale, i.e. without consideration of microscopic details, such as the opening of a particular ion channel in the microsecond scale. For example, this approach could simplify and speed up the investigation of propagation problems such as infarct's scars at the heart scale and its components (ventricles, atria, etc). This remains true only when the phenomena under study needs not take into account more complicated dynamics than the four AP phases. Our method should also be sufficient for direct simulations of electrocardiograms, and hopefully much more. Finally, it could open the door to many other applications like the inverse problem. It is just then a matter of setting the parameters of a well-known simple model instead of developing and coupling complex ionic models for several regions of the heart. 
Acknowledgements. This work was supported by NSERC and FQRNT Graduate Scholarships to the first author and a NSERC Discovery Grant to the second author. The authors wish to thank Yves Coudière from INRIA Bordeaux Sud-Ouest for fruitful comments.

\section{REFERENCES}

[1] R.R. Aliev and A.V. Panfilov, A simple two-variable model of cardiac excitation. Chaos Soliton. Fract. 7 (1996) $293-301$.

[2] M. Beck, C.K.R.T. Jones, D. Schaeffer and M. Wechselberger, Electrical Waves in a One-Dimensional Model of Cardiac Tissue. SIAM J. Appl. Dynam. Syst. 7 (2008) 1558-1581.

[3] G. W. Beeler and H. Reuter, Reconstruction of the action potential of ventricular myocardial fibres. J. Physiol. 268 (1977) $177-210$.

[4] M. Boulakia, M. Fernàndez, J.-F. Gerbeau and N. Zemzemi, Towards the numerical simulation of electrocardiograms, in Functional Imaging and Modeling of the Heart, vol. 4466 of Lect. Notes Comput. Sci., edited by F. Sachse and G. Seemann. Springer, Berlin/Heidelberg (2007) 240-249.

[5] N. F. Britton, Essential Mathematical Biology. Springer Undergrad. Math. Series (2005).

[6] J.W. Cain, Taking math to the heart: Mathematical challenges in cardiac electrophysiology. Notices of the AMS $\mathbf{5 8}$ (2011) $542-549$.

[7] R.H. Clayton and A.V. Panfilov, A guide to modelling cardiac electrical activity in anatomically detailed ventricles. Prog. Biophys. Mol. Bio. 96 (2008) 19-43.

[8] P. Colli Franzone, L. Guerri and S. Rovida, Wavefront propagation in an activation model of the anisotropic cardiac tissue: asymptotic analysis and numerical simulations. J. Math. Biol. 28 (1990) 121-176. DOI: 10.1007/BF00163143.

[9] B. Deng, The existence of infinitely many traveling front and back waves in the Fitzhugh - Nagumo equations. SIAM J. Math. Anal. 22 (1991) 1631-1650.

[10] K. Djabella, M. Landau and M. Sorine, A two-variable model of cardiac action potential with controlled pacemaker activity and ionic current interpretation. 46th IEEE Conf. Decis. Control (2007) 5186-5191.

[11] E.G. Tolkacheva, D.G. Schaeffer, D.J. Gauthier and C.C. Mitchell, Analysis of the Fenton-Karma model through an approximation by a one-dimensional map. Chaos 12 (2002) 1034-1042 .

[12] F. Fenton and A. Karma, Vortex dynamics in three-dimensional continuous myocardium with fiber rotation: Filament instability and fibrillation. Chaos 8 (1998) 20-47.

[13] R.A. FitzHugh, Impulses and physiological states in theoretical models of nerve membrane. Biophys. J. 1 (1961) 445-466.

[14] S. Hastings, Single and multiple pulse waves for the Fitzhugh-Nagumo equations. SIAM J. Appl. Math. 42 (1982) $247-260$.

[15] A.L. Hodgkin and A.F. Huxley, A quantitative description of membrane current and its application to conduction and excitation in nerve. J. Physiol. 117 (1952) 500-544.

[16] J. Keener and J. Sneyd, Mathematical Physiology. Springer (2004).

[17] J.P. Keener, Modeling electrical activity of cardiac cells, Two variable models, Mitchell-Schaeffer revised. Available at www.math.utah.edu/ keener/lectures/ionic_models/Two_variable_models.

[18] J.P. Keener, An eikonal-curvature equation for action potential propagation in myocardium. J. Math. Biol. 29 (1991) 629-651. DOI : $10.1007 / \mathrm{BF} 00163916$.

[19] K.H. Ten Tusscher, D. Noble, P.J. Noble and A.V. Panfilov, A model for human ventricular tissue. Am. J. Physiol. Heart Circ. Physiol. 286 (2004) H1973-H1589.

[20] R. Killmann, P. Wach and F. Dienstl, Three-dimensional computer model of the entire human heart for simulation of reentry and tachycardia: gap phenomenon and Wolff-Parkinson-White syndrome. Basic Res. Cardiol. 86 (1991) $485-501$.

[21] C.H. Luo and Y. Rudy, A dynamic model of the cardiac ventricular action potential: I. simulations of ionic currents and concentration changes. Circ. Res. 74 (1994) 1071-1096.

[22] C. Mitchell and D. Schaeffer, A two-current model for the dynamics of cardiac membrane. Bull. Math. Bio. 65 (2003) $767-793$.

[23] B.R. Munson, D.F. Young and T.H. Okiishi, Fundamentals of Fluid Mechanics. Wiley and Sons (2001).

[24] J. Nagumo, S. Arimoto and S. Yoshizawa, An active pulse transmission line simulating nerve axon. Proc. IRE. 50 (1962) 2061-2070.

[25] D. Noble, A modification of the Hodgkin-Huxley equations applicable to purkinje fibre action and pacemaker potentials. J. Physiol. 160 (1962) 317-352.

[26] C. Pierre, Modélisation et simulation de l'activité électrique du coeur dans le thorax, analyse numérique et méthodes de volumes finis. PhD thesis, University of Nantes (2005).

[27] J. Relan, M. Sermesant, H. Delingette, M. Pop, G.A. Wright and N. Ayache, Quantitative comparison of two cardiac electrophysiology models using personalisation to optical and $\mathrm{mr}$ data, in Proc. Sixth IEEE Int. Symp. Biomed. Imaging 2009 (ISBI'09).

[28] J. Relan, M. Sermesant, M. Pop, H. Delingette, M. Sorine, G.A. Wright and N. Ayache, Parameter estimation of a 3d cardiac electrophysiology model including the restitution curve using optical and MR data, in World Congr. on Med. Phys. and Biomed. Eng., WC 2009, München (2009). 
[29] D. Schaeffer, J. Cain, D. Gauthier, S. Kalb, R. Oliver, E. Tolkacheva, W. Ying and W. Krassowska, An ionically based mapping model with memory for cardiac restitution. Bull. Math. Bio. 69 (2007) 459-482. DOI: 10.1007/s11538-006-9116-6.

[30] D. Schaeffer, W. Ying and X. Zhao, Asymptotic approximation of an ionic model for cardiac restitution. Nonlinear Dyn. 51 (2008) 189-198. DOI: 10.1007/s11071-007-9202-9.

[31] M. Sermesant, Y. Coudière, V. Moreau Villéger, K.S. Rhode, D.L.G. Hill and R. Ravazi, A fast-marching approach to cardiac electrophysiology simulation for XMR interventional imaging, in Proc. of MICCAI'05, vol. 3750 of Lect. Notes Comput. Sci., Palm Springs, California. Springer Verlag (2005) 607-615.

[32] J. Sundnes, G.T. Lines, X. Cai, B.F. Nielsen, K.-A. Mardal and A. Tveito, Computing the Electrical Activity in the Heart. Springer, Monogr. Comput. Sci. Eng. 1 (2006). 